\title{
Stability Analysis of Polytopic Discontinuous Galerkin Approximations of the Stokes Problem with Applications to Fluid-Structure Interaction Problems
}

\author{
Paola F. Antonietti ${ }^{1} \cdot$ Lorenzo Mascotto $^{2,3} \cdot$ Marco Verani $^{1} \cdot$ Stefano Zonca ${ }^{1}$ ib
}

Received: 1 December 2020 / Revised: 28 September 2021 / Accepted: 29 September 2021

(c) The Author(s), under exclusive licence to Springer Science+Business Media, LLC, part of Springer Nature 2021

\begin{abstract}
We present a stability analysis of the Discontinuous Galerkin method on polygonal and polyhedral meshes (PolyDG) for the Stokes problem. In particular, we analyze the discrete inf-sup condition for different choices of the polynomial approximation order of the velocity and pressure approximation spaces. To this aim, we employ a generalized inf-sup condition with a pressure stabilization term. We also prove a priori $h p$-version error estimates in suitable norms. We numerically check the behaviour of the inf-sup constant and the order of convergence with respect to the mesh configuration, the mesh-size, and the polynomial degree. Finally, as a relevant application of our analysis, we consider the PolyDG approximation for a 2D fluid-structure interaction problem and we numerically explore the stability properties of the method.
\end{abstract}

Keywords Discontinuous Galerkin · Polygonal and polyhedral meshes · Fluid-structure interaction

Mathematics Subject Classification 65M60 - 74F10 - 65N12

PFA, LM, MV and SZ are member of the INdAM Research group GNCS and this work is partially funded by INDAM-GNCS. PFA, MV and SZ have been partially funded by the PRIN Italian research grant $n$. 201744KLJL funded by MIUR. LM acknowledges the support of the Austrian Science Fund (FWF) project P33477.

$凶$ Stefano Zonca

stefano.zonca@polimi.it

Paola F. Antonietti

paola.antonietti@polimi.it

Lorenzo Mascotto

lorenzo.mascotto@unimib.it

Marco Verani

marco.verani@polimi.it

1 MOX, Dipartimento di Matematica, Politecnico di Milano, Milan, Italy

2 Dip. di Matematica e Applicazioni, Universitá degli Studi di Milano-Bicocca, Milan, Italy

3 Fakultät für Mathematik, Universität Wien, Wien, Austria 


\section{Introduction}

It is well known that a crucial aspect involving the stability of the numerical scheme associated with the Stokes problem is the inf-sup condition that establishes a constraint in the choice of the velocity and pressure discrete spaces; see, e.g, [13,19]. This aspect, in the context of polygonal methods, is still under investigation and only few results are present in the literature; see, e.g., [2,20,29,31,33,65,68].

The Discontinuous Galerkin (DG) method handles meshes with elements of general shape and has proved to be suited for the approximation of fluid and structure models, possibly involving moving domains, see, e.g. [35,59]. The discrete inf-sup condition for DG methods has been analyzed in the following works. In [29], the Local DG method for the Stokes problem is formulated in a conservative way, by introducing the stress as unknown. Here, meshes with hanging nodes and elements of different shape are considered, provided that they are affinely-equivalent to an element of a fixed set of reference elements. Moreover, an inf-sup condition and optimal order estimates are proven, when the pair of polynomials of degree $k$ and $k-1$ is chosen for the velocity and pressure spaces. However, the formulation requires a stability term for both the velocity and the pressure. In [54], the inf-sup condition is proven for a pressure stabilized formulation on hexahedral meshes allowing hanging nodes, when the pair $\mathbb{Q}_{k}-\mathbb{Q}_{k}$ is chosen. In [31,32], the authors show the inf-sup condition for equalorder approximation $\mathbb{P}_{k}$ for both the velocity and pressure in the case of a pressure stabilized formulation on meshes consisting of elements of various shape, provided that each element is affinely-equivalent to one in a fixed set of reference elements, and admitting hanging nodes. In [53], the authors propose a mixed DG formulation without pressure stabilization for the Stokes problem and show a priori error estimates. The inf-sup condition is proven for the pair of spaces $\mathbb{Q}_{k}-\mathbb{Q}_{k-1}$ on tensor product meshes, possibly with hanging nodes. In [63], the inf-sup condition is proven for the pairs of spaces $\mathbb{Q}_{k}-\mathbb{Q}_{k-1}$ and $\mathbb{Q}_{k}-\mathbb{Q}_{k-2}$ without any pressure stabilization on quadrilateral and hexahedral meshes with hanging nodes; see also $[52,57,64]$. Numerical tests showing the dependence of the inf-sup constants are performed for the pairs of spaces $\mathbb{Q}_{k}-\mathbb{Q}_{k^{\prime}}$, with $k^{\prime}=k, k-1, k-2$. In [45], the inf-sup condition is proven on triangular and tetrahedral meshes without any pressure stabilization term for the pair of spaces $\mathbb{P}_{k}-\mathbb{P}_{k-1}$ employing the Brezzi-Douglas-Marini spaces. In [41], the pair of spaces $\mathbb{P}_{k}-\mathbb{P}_{k-1}$ with the Crouzeix-Raviart elements is used to prove the inf-sup condition on triangular meshes.

In this work, we consider the Discontinuous Galerkin method on polygonal and polyhedral grids (PolyDG) that extends the standard DG method to polytopic meshes; see, e.g., [4$7,11,26,28,66]$. In this framework, we study the discrete stability and well-posedness for the Stokes problem, by presenting an analysis that covers at once the two- and three- dimensional cases. Under suitable assumptions, we prove that the inf-sup constant is independent of the mesh size. Notwithstanding, it is not robust with respect to the polynomial degree and this restriction propagates to the convergence analysis, with a deterioration of the convergence in terms of the polynomial degree. However, we provide numerical evidence that the discrete infsup constant has a much milder dependence on the polynomial degree in practice. Moreover, the mesh assumptions seem to be too restrictive and, in fact, the method results to be infsup stable also for pathological configurations. In the two-dimensional case, we numerically assess the robustness of the inf-sup constant with respect to the mesh size and the polynomial degree for different types of mesh elements, including elements with degenerating edges, and we numerically estimate the order of convergence to the mesh size and the polynomial degree. 
Besides, with the aim of further exploring the relevance of our stability analysis, we consider a fluid-structure interaction (FSI) problem where both the Stokes and the elastodynamics equations are solved based on employing the PolyDG method. In fact, it is well known that the study of FSI problems is of paramount importance in many engineering and biomedical applications; see, e.g., $[17,40,47,51,61,67]$, where a fluid, for instance modeled via the Stokes equations, interacts with a structure, modeled via the elastodynamics equations. Indeed, to correctly model such problems, ad-hoc techniques are mandatory to deal with the movement of the structures. A classical strategy to overcome this issue is the employment of the Arbitrary Lagrangian Eulerian (ALE) approach. It consists in deforming the fluid grid according to the structure displacement, yet maintaining a "honouring" mesh at the fluid-structure interface and generating an arbitrary deformation of the elements in the interior of the fluid mesh; see, e.g., [34,46,60]. Another way that preserves the alignment of the fluid and structure grids at the interface is to use approaches based on remeshing and mesh-adaptation techniques; see, e.g., [16,62]. A different category of approaches are based on employing unfitted meshes that allow to keep the fluid grid fixed in time, while the structure mesh is free to move; see, e.g., $[1,3,14,15,22,30,38,39,42,43,49,50,69]$. Often, this requires the handling of polygonal and polyhedral elements appearing in the fluid mesh, e.g., due to the intersection between the fluid and structure elements, and in the solid mesh, e.g., due to the presence of hanging nodes; see, e.g., [8,12]. For this kind of approaches, it is mandatory that the underlining discretization methods can robustly and efficiently support meshes made of arbitrarily shaped elements. In this respect, a deep understanding of the stability properties of the numerical scheme with respect to possibly pathological meshes is of crucial importance.

The paper is organized as follows. Section 2 introduces the transient Stokes problem and its PolyDG approximation. In Sect. 3, we prove the well-posedness of the PolyDG approximation of the (stationary) Stokes problem, with a particular emphasis to the discrete inf-sup condition. In particular, in Sect. 4 we estimate the discrete inf-sup constant and numerically evaluate it for different choices of the discrete velocity and pressure spaces and for different grids. Section 5 is devoted to the proof of a priori error estimates of the Stokes problem. In Sect. 6, we introduce a fluid-structure interaction problem and we present its fully-discrete PolyDG approximation. In Sect. 7 we show some numerical results for the Stokes and FSI problems. Finally, in Sect. 8, we draw some conclusions.

In the sequel, the notation $\lesssim$ and $\gtrsim$ means that the inequalities are valid up to multiplicative constants that are independent of the discretization parameters, i.e., the mesh size and the polynomial approximation degree but might depend on the physical parameters of the underlying problem as well as on the shape-regularity constants of the underlying mesh; cf. Sect. 3.1 below.

\section{The Transient Stokes Problem}

Having in mind the PolyDG discretization of FSI problems as a reference application, in this section we consider the transient Stokes problem which reads as follows: given a final time $T>0, f$ a (regular) forcing term, and $\mu$ and $\rho$ the viscosity and density of the fluid, find the velocity $\boldsymbol{u}=\boldsymbol{u}(t)$ and the pressure $p=p(t)$ such that, for all $t \in(0, T]$,

$$
\begin{array}{ll}
\rho \partial_{t} \boldsymbol{u}-\mu \Delta \boldsymbol{u}+\nabla p=\boldsymbol{f} & \text { in } \Omega, \\
\nabla \cdot \boldsymbol{u}=0 & \text { in } \Omega,
\end{array}
$$




$$
\boldsymbol{u}=0 \quad \text { on } \partial \Omega \text {. }
$$

Problem (1) is supplemented with sufficiently regular initial conditions $\boldsymbol{u}(\boldsymbol{x}, 0)=\boldsymbol{u}^{0}(\boldsymbol{x})$ in $\Omega$. To guarantee the well-posedness of the problem, we prescribe that $p \in L_{0}^{2}(\Omega)$, where $L_{0}^{2}(\Omega)$ is the space of $L^{2}(\Omega)$ functions with zero average over $\Omega$.

We introduce the functional spaces

$$
\boldsymbol{V}=\left\{\boldsymbol{v} \in\left[H^{1}(\Omega)\right]^{d}, d=2,3, \text { such that }\left.\boldsymbol{v}\right|_{\partial \Omega}=0\right\}
$$

and $Q=L_{0}^{2}(\Omega)$ and endow them with the norms

$$
\|\boldsymbol{v}\|_{\boldsymbol{V}}:=\left\|\mu^{\frac{1}{2}} \nabla \boldsymbol{v}\right\|_{L^{2}(\Omega)} \quad \text { and } \quad\|q\|_{Q}:=\|q\|_{L^{2}(\Omega)} .
$$

The weak formulation of problem (1) reads as follows: find $(\boldsymbol{u}, p) \in \boldsymbol{V} \times Q$, such that, for all $t \in(0, T]$,

$$
\left(\rho \partial_{t} \boldsymbol{u}, \boldsymbol{v}\right)_{\Omega}+a(\boldsymbol{u}, \boldsymbol{v})+b(p, \boldsymbol{v})-b(q, \boldsymbol{u})=(\boldsymbol{f}, \boldsymbol{v})_{\Omega} \quad \forall(\boldsymbol{v}, q) \in \boldsymbol{V} \times Q,
$$

where

$a: \boldsymbol{V} \times \boldsymbol{V} \rightarrow \mathbb{R}, \quad a(\boldsymbol{u}, \boldsymbol{v})=\int_{\Omega} \mu \nabla \boldsymbol{u}: \nabla \boldsymbol{v}, \quad b: Q \times \boldsymbol{V} \rightarrow \mathbb{R}, \quad b(p, \boldsymbol{v})=-\int_{\Omega} p \nabla \cdot \boldsymbol{v}$,

and $(\cdot, \cdot)_{\Omega}$ denotes the $L^{2}$-inner product over the domain $\Omega$. It is well-known that the bilinear form $b(\cdot, \cdot)$ satisfies a continuous inf-sup condition; see, e.g., [13]. More precisely, there exists a universal positive constant depending only on $\Omega$ such that, to all $q \in L_{0}^{2}(\Omega)$, we associate a function $\boldsymbol{v}_{q} \in \boldsymbol{V}$ satisfying $\nabla \cdot \boldsymbol{v}_{q}=q$ and

$$
\beta\left\|\boldsymbol{v}_{q}\right\|_{\boldsymbol{V}} \leq\|q\|_{L^{2}(\Omega)} .
$$

\subsection{PolyDG Semi-Discrete Approximation of the Transient Stokes Problem}

First, we introduce the necessary notation and key analytical results required for the definition and analysis of PolyDG semi-discrete approximation of the transient Stokes problem.

We introduce a mesh $\mathcal{T}_{h}$ composed of polytopic elements $K$ of arbitrary shape. We indicate with $h_{K}$ the diameter of the element $K$. We define an interface to be either the intersection of the $(d-1)$-dimensional facets of two neighboring elements or the intersection of the $(d-1)$ dimensional facets of an element with the boundary of $\Omega$. When $d=2$, interfaces coincide with edges and consist of line segments; in presence of hanging nodes, a single line segment can contain more than one interface. When $d=3$, we assume that each interface consists of a general planar polygon that we assume that can be further decomposed into a set of co-planar triangles, denoted as faces. With this notation, we collect all the $(d-1)$-dimensional faces in the set $\mathcal{F}_{h}$, i.e., any face $F \in \mathcal{F}_{h}$ is always defined as a set of $(d-1)$-dimensional simplices (line segments or triangles); cf. [27,28]. We also decompose the faces $\mathcal{F}_{h}$ into $\mathcal{F}_{h}=\mathcal{F}_{h}^{i} \cup \mathcal{F}_{h}^{b}$, where $\mathcal{F}_{h}^{i}$ denotes the set of interior faces and $\mathcal{F}_{h}^{b}$ denotes the set of boundary faces. To avoid technicalities, in the following we assume that $\rho$ and $\mu$ are piecewise constant over the mesh.

For given integers $\ell, m \geq 1$, we introduce the DG finite element spaces

$$
\begin{aligned}
& \mathbf{V}_{h}^{\ell}=\left\{\boldsymbol{v} \in\left[L^{2}(\Omega)\right]^{d}:\left.\boldsymbol{v}\right|_{K} \in\left[\mathcal{P}^{\ell}(K)\right]^{d} \forall K \in \mathcal{T}_{h}\right\}, \\
& Q_{h}^{m}=\left\{q \in L_{0}^{2}(\Omega):\left.q\right|_{K} \in \mathcal{P}^{m}(K) \forall K \in \mathcal{T}_{h}\right\},
\end{aligned}
$$

where $\mathcal{P}^{k}(K), k \geq 1$, denotes the space of polynomials defined over the element $K \in \mathcal{T}_{h}$ of total degree at most $k$. In practice, the shape functions and the degrees of freedom are 
directly generated on the physical element $K \in \mathcal{T}_{h}$ with the "bounding box" technique; see, e.g., [28].

On any interior face $F \in \mathcal{F}_{h}^{i}$ and for sufficiently regular scalar, vector-valued and symmetric tensor-valued functions $q, \boldsymbol{v}$ and $\boldsymbol{T}$, respectively, we define the average and jump operators as

$$
\begin{array}{ll}
\{\boldsymbol{v}\}=\frac{1}{2}\left(\boldsymbol{v}^{+}+\boldsymbol{v}^{-}\right), & \llbracket q \rrbracket=q^{+} \boldsymbol{n}^{+}+q^{-} \boldsymbol{n}^{-}, \\
\{\boldsymbol{T}\}=\frac{1}{2}\left(\boldsymbol{T}^{+}+\boldsymbol{T}^{-}\right), & \llbracket \boldsymbol{v} \rrbracket=\boldsymbol{v}^{+} \odot \boldsymbol{n}^{+}+\boldsymbol{v}^{-} \odot \boldsymbol{n}^{-},
\end{array}
$$

where $q^{ \pm}, \boldsymbol{v}^{ \pm}$and $\boldsymbol{T}^{ \pm}$denote the traces of $q, \boldsymbol{v}$ and $\boldsymbol{T}$ on $F$ taken within the interior of $K^{ \pm}$ and where $\boldsymbol{v} \odot \boldsymbol{n}=\left(\boldsymbol{v} \boldsymbol{n}^{T}+\boldsymbol{n} \boldsymbol{v}^{T}\right) / 2$. The jump $\llbracket \boldsymbol{v} \rrbracket$ is a symmetric tensor-valued function. On a boundary face $F \in \mathcal{F}_{h}^{b}$, we set analogously

$$
\begin{array}{lll}
\{\boldsymbol{v}\}=\boldsymbol{v}, & \llbracket q \rrbracket=q \boldsymbol{n}, \\
\{\boldsymbol{T}\}=\boldsymbol{T}, & \llbracket \boldsymbol{v} \rrbracket=\boldsymbol{v} \odot \boldsymbol{n} .
\end{array}
$$

We also introduce the $L^{2}$-inner products over a domain $Z \subset \mathbb{R}^{d}, d=1,2,3$, and a face $F \in \mathcal{F}_{h}$ with the shorthand notation $(\cdot, \cdot)_{Z}$ and $(\cdot, \cdot)_{F}$, respectively.

Given $s>1 / 2$, associated with any mesh $\mathcal{T}_{h}$, we introduce the broken Sobolev space $H^{s}\left(\mathcal{T}_{h}\right)=\left\{v \in L^{2}(\Omega)|v|_{K} \in H^{s}(K)\right.$ for all $\left.K \in \mathcal{T}_{h}\right\}$. The standard Dirichlet trace operator is well defined on the skeleton of the mesh for functions in $H^{s}\left(\mathcal{T}_{n}\right)$. We define the stabilization functions $\sigma_{v} \in L^{\infty}\left(\mathcal{F}_{h}\right)$ and $\sigma_{p} \in L^{\infty}\left(\mathcal{F}_{h}\right)$ as follows.

Definition 1 We define the functions $\sigma_{v}: \mathcal{F}_{h} \rightarrow \mathbb{R}$ and $\sigma_{p}: \mathcal{F}_{h}^{i} \rightarrow \mathbb{R}$ as

$$
\left.\sigma_{v}\right|_{F}=\left\{\left.\begin{array}{ll}
\gamma_{v} \max _{K^{+}, K^{-}}\left\{\frac{\ell^{2} \mu}{h_{K}}\right\} & F \in \mathcal{F}_{h}^{i}, \\
\gamma_{v} \frac{\ell^{2} \mu}{h_{K}} & F \in \mathcal{F}_{h}^{b},
\end{array} \quad \sigma_{p}\right|_{F}=\gamma_{p} \min _{K^{+}, K^{-}}\left\{\frac{h_{K}}{m}\right\} \quad F \in \mathcal{F}_{h}^{i},\right.
$$

where $\gamma_{v}$ and $\gamma_{p}$ are two universal positive constants, and $\ell$ and $m$ denote the polynomial approximation degrees for the velocity and the pressure, respectively.

Next, we introduce three bilinear forms that are instrumental for the construction of the DG method. More precisely, we define

$$
\begin{aligned}
a_{h}(\boldsymbol{u}, \boldsymbol{v})= & \int_{\Omega} \mu \nabla_{h} \boldsymbol{u}: \nabla_{h} \boldsymbol{v}-\sum_{F \in \mathcal{F}_{h}} \int_{F} \mu\left\{\nabla_{h} \boldsymbol{u}\right\}: \llbracket \boldsymbol{v} \rrbracket \\
& -\sum_{F \in \mathcal{F}_{h}} \int_{F} \mu \llbracket \boldsymbol{u} \rrbracket:\left\{\nabla_{h} \boldsymbol{v}\right\}+\sum_{F \in \mathcal{F}_{h}} \int_{F} \sigma_{v} \llbracket \boldsymbol{u} \rrbracket: \llbracket \boldsymbol{v} \rrbracket, \\
b_{h}(p, \boldsymbol{v})= & -\int_{\Omega} p \nabla_{h} \cdot \boldsymbol{v}+\sum_{F \in \mathcal{F}_{h}} \int_{F}\{p \boldsymbol{I}\}: \llbracket \boldsymbol{v} \rrbracket, \\
s_{h}(p, q)= & \sum_{F \in \mathcal{F}_{h}^{i}} \int_{F} \sigma_{p} \llbracket p \rrbracket \cdot \llbracket q \rrbracket,
\end{aligned}
$$

where $\nabla_{h}$ is the piecewise broken gradient operator. The bilinear form $s_{h}(p, q)$ represents the pressure stabilization term and $\sigma_{p}$ plays the role of penalty parameter. Given $\boldsymbol{f} \in\left[L^{2}(\Omega)\right]^{d}$, 
the semi-discrete PolyDG approximation of (2) reads as follows: for any $t \in(0, T]$, find $\left(\boldsymbol{u}_{h}, p_{h}\right) \in \mathbf{V}_{h}^{\ell} \times Q_{h}^{m}$ such that

$$
\left(\rho \partial_{t} \boldsymbol{u}_{h}, \boldsymbol{v}_{h}\right)_{\Omega}+a_{h}\left(\boldsymbol{u}_{h}, \boldsymbol{v}_{h}\right)+b_{h}\left(p_{h}, \boldsymbol{v}_{h}\right)-b_{h}\left(q_{h}, \boldsymbol{u}_{h}\right)+s_{h}\left(p_{h}, q_{h}\right)=\left(\boldsymbol{f}, \boldsymbol{v}_{h}\right)_{\Omega}
$$

for all $\left(\boldsymbol{v}_{h}, q_{h}\right) \in \mathbf{V}_{h}^{\ell} \times Q_{h}^{m}$.

\section{Well-Posedness of the Stationary Stokes Problem}

In this section, we prove the well-posedness of problem (5) in the stationary case making use of the Banach-Nečas-Babuška theorem. To this aim, we first introduce

$$
\begin{gathered}
\mathcal{B}_{h}((\boldsymbol{u}, p),(\boldsymbol{v}, q))=a_{h}(\boldsymbol{u}, \boldsymbol{v})+b_{h}(p, \boldsymbol{v})-b_{h}(q, \boldsymbol{u})+s_{h}(p, q), \\
\quad F((\boldsymbol{v}, q))=(\boldsymbol{f}, \boldsymbol{v})_{\Omega},
\end{gathered}
$$

and re-write the stationary discrete Stokes problem as follows: find $\left(\boldsymbol{u}_{h}, p_{h}\right) \in \mathbf{V}_{h}^{\ell} \times Q_{h}^{m}$ such that

$$
\mathcal{B}_{h}\left(\left(\boldsymbol{u}_{h}, p_{h}\right),\left(\boldsymbol{v}_{h}, q_{h}\right)\right)=F\left(\left(\boldsymbol{v}_{h}, q_{h}\right)\right) \quad \forall\left(\boldsymbol{v}_{h}, q_{h}\right) \in \mathbf{V}_{h}^{\ell} \times Q_{h}^{m} .
$$

On the product space $\mathbf{V}_{h}^{\ell} \times Q_{h}^{m}$, we define the norm

$$
\left\|\left(\boldsymbol{v}_{h}, q_{h}\right)\right\|_{\mathrm{E}}^{2}=\left\|\boldsymbol{v}_{h}\right\|_{\mathbf{V}_{h}^{\ell}}^{2}+\left\|q_{h}\right\|_{Q_{h}^{m}}^{2} \quad \forall\left(\boldsymbol{v}_{h}, q_{h}\right) \in \mathbf{V}_{h}^{\ell} \times Q_{h}^{m},
$$

where

$$
\begin{aligned}
\left\|\boldsymbol{v}_{h}\right\|_{\mathbf{V}_{h}^{\ell}}^{2} & =\sum_{K \in \mathcal{T}_{k}}\left\|\mu^{1 / 2} \nabla_{h} \boldsymbol{v}_{h}\right\|_{L^{2}(K)}^{2}+\left\|\sigma_{v}^{1 / 2} \llbracket \boldsymbol{v}_{h} \rrbracket\right\|_{L^{2}\left(\mathcal{F}_{h}\right)}^{2} \quad \\
\left\|q_{h}\right\|_{Q_{h}^{m}}^{2} & =\left\|q_{h}\right\|_{L^{2}(\Omega)}^{2}+\left|q_{h}\right|_{\jmath}^{2}, \quad\left|q_{h}\right|_{\jmath}^{2}=s_{h}\left(q_{h}, q_{h}\right) \quad \forall \mathbf{V}_{h}^{\ell},
\end{aligned}
$$

Before presenting the theoretical analysis, we introduce some mesh assumptions and technical results that will be needed in the forthcoming analysis.

\subsection{Mesh Assumptions and Preliminary Results}

Following [5,24,28], we introduce the notion of a family of polytopic-regular meshes $\mathcal{T}_{h}$. To this end, we write $\tau_{K_{F}}$ to denote a $d$-dimensional simplex contained in $K \in \mathcal{T}_{h}$, which shares a specific face $F \subset \partial K, F \in \mathcal{F}_{h}$.

Definition 2 A family of polytopic meshes $\left\{\mathcal{T}_{h}\right\}_{h}$ is said to be polytopic-regular if, for any $h$ and $K \in \mathcal{T}_{h}$, there exists a set of non-overlapping (not necessarily shape-regular) $d$ dimensional simplices $\left\{\tau_{K_{F}}\right\}_{F \subset \partial K}$ contained in $K$, such that, for all faces $F \subset \partial K, h_{K} \lesssim$ $\frac{\left|\tau_{K_{F}}\right|}{|F|}$.

This definition is very general as it does not require any restriction on either the number of faces per element or their relative measure. In particular, it allows the size of a face $F \subset \partial K$ to be arbitrarily small compared to the diameter of the element $h_{K}$ it belongs to, provided that the height of the corresponding simplex $\tau_{K_{F}}$ is comparable to $h_{K}$; cf. [27] for more details.

In order to state suitable approximation results, cf. Lemmata 2 and 3 below and [28], we introduce a shape-regular covering $\mathcal{T}_{h}^{\#}=\left\{T_{K}\right\}$ of $\mathcal{T}_{h}$ defined as a set of shape-regular 
$d$-dimensional simplices $T_{K}$, such that, for each $K \in \mathcal{T}_{h}$, there exists a $T_{K} \in \mathcal{T}_{h}^{\#}$ such that $K \subsetneq T_{K}$.

We introduce the following assumption on the mesh $\mathcal{T}_{h}$; cf. [27,28].

Assumption 3.1 Given $\left\{\mathcal{T}_{h}\right\}_{h}, h>0$, we assume that the following properties are uniformly satisfied:

A.1 $\mathcal{T}_{h}$ is uniformly polytopic-regular in the sense of Definition 2;

A.2 we assume that there exists a shape-regular covering $\mathcal{T}_{h}^{\#}$ of $\mathcal{T}_{h}$ such that, for each pair $K \in \mathcal{T}_{h}, \mathcal{K} \in \mathcal{T}_{h}^{\#}$ with $K \subset \mathcal{K}$, the following properties are fulfilled: $\left.i\right) h_{\mathcal{K}} \lesssim h_{K}$ and ii) $\max _{K \in \mathcal{T}_{h}} \operatorname{card}\left\{K^{\prime} \in \mathcal{T}_{h}: K^{\prime} \cap \mathcal{K} \neq \emptyset, \mathcal{K} \in \mathcal{T}_{h}^{\#}, K \subset \mathcal{K}\right\} \lesssim 1$;

$A .3$ for any pair of elements $K, K^{\prime} \in \mathcal{T}_{h}$ sharing a face $F \in \mathcal{F}_{h}$, we have: $h_{K} \lesssim h_{K^{\prime}}$ and $h_{K^{\prime}} \lesssim h_{K}$.

The local bounded variation hypothesis A.3 has been introduced to avoid technicalities. Removing this hypothesis would render some forthcoming results more complicated and with a loss of local bounds; see the proofs of Proposition 1 and Corollary 1.

The following trace-inverse inequality is valid; see, e.g., [27, Lemma 11].

Lemma 1 (Polynomial trace inverse inequality) Let Assumption A.1 be valid. For each $K \in$ $\mathcal{T}_{h}$, the following trace-inverse inequality is valid:

$$
\|v\|_{L^{2}(\partial K)}^{2} \lesssim \frac{\ell^{2}}{h_{K}}\|v\|_{L^{2}(K)}^{2} \quad \forall v \in \mathcal{P}^{\ell}(K), \quad \ell \geq 1 .
$$

Let $\mathcal{E}: H^{s}(\Omega) \rightarrow H^{s}\left(\mathbb{R}^{d}\right), s \geq 0$, be the Stein extension operator for Sobolev spaces on Lipschitz domains introduced in [56, Chapter 3]. The operator $\mathcal{E}$ satisfies the following property: given a domain $\Omega$ with Lipschitz boundary, for all $q \in H^{s}(\Omega)$,

$$
\left.\mathcal{E}(q)\right|_{\Omega}=q, \quad\|\mathcal{E} q\|_{H^{s}\left(\mathbb{R}^{d}\right)} \lesssim\|q\|_{H^{s}(\Omega)} .
$$

For vector-valued functions, the Stein extension operator is defined component-wise. We recall the following approximation result; see, e.g., [24,27,28] for a detailed proof, which generalizes the standard arguments for standard geometries $[9,10]$.

Lemma 2 (Best polynomial approximation in Sobolev norms) Let Assumption A.2 be valid. Given the Stein extension operator $\mathcal{E}$ in $(10)$, let $v \in L^{2}(\Omega)$ be such that $\left.(\mathcal{E} v)\right|_{\mathcal{K}} \in H^{r}(\mathcal{K})$, for some $r \geq 0$. Then, there exists a sequence of polynomial approximations $\Pi_{K}^{\ell} v \in \mathcal{P}_{\ell}(K)$ of $v, K \in \mathcal{T}_{h}$ and $\ell \in \mathbb{N}$ of $v$ satisfying

$$
\left\|v-\Pi_{K}^{\ell} v\right\|_{H^{q}(K)} \lesssim \frac{h_{K}^{\min \{\ell+1, r\}-q}}{\ell^{r-q}}\|\mathcal{E} v\|_{H^{r}(\mathcal{K})}, \quad 0 \leq q \leq r,
$$

where $\mathcal{K} \in \mathcal{T}_{h}^{\#}$ is the $d$-simplex of $\mathcal{T}_{h}^{\#}$ such that $K \subset \mathcal{K}$.

Based on employing the above result, we define the global polynomial approximation operator $\Pi^{\ell} v$ as $\left.\left(\Pi^{\ell} v\right)\right|_{K}=\Pi_{K}^{\ell}\left(\left.v\right|_{K}\right) \quad \forall K \in \mathcal{T}_{h}$. For vector-valued functions, the operators $\Pi_{K}^{\ell}$ and $\Pi^{\ell}$ are defined component-wise and are still denoted by $\Pi_{K}^{\ell}$ and $\Pi^{\ell}$, respectively. We have the following approximation bound in the energy norm (9).

Lemma 3 (Best polynomial approximation in the DG norm) Let Assumption 3.1 be valid. Let $\boldsymbol{v} \in\left[L^{2}(\Omega)\right]^{d}$ be such that, for some $r \geq 1,\left.(\mathcal{E} v)\right|_{\mathcal{K}} \in\left[H^{r}(\mathcal{K})\right]^{d}$ for all $\mathcal{K} \in \mathcal{T}_{h}^{\#}, r \geq 1$. Then, we have

$$
\left\|\boldsymbol{v}-\Pi^{\ell} \boldsymbol{v}\right\|_{\mathbf{V}_{h}^{\ell}}^{2} \lesssim \sum_{K \in \mathcal{T}_{h}} \frac{h_{K}^{2(\min \{\ell+1, r\}-1)}}{\ell^{2(r-1)-1}}\|\mathcal{E} \boldsymbol{v}\|_{H^{r}(\mathcal{K})}^{2}
$$


Finally, we recall the following continuity and coercivity bounds for the bilinear form $a_{h}(\cdot, \cdot)$. The proof is based upon employing the trace-inverse estimate in Lemma 1 and standard arguments for DG methods; see, e.g., [27].

Lemma 4 (Coercivity and continuity of $a_{h}(\cdot, \cdot)$ ) Let Assumption 3.1 be valid. Then, we have

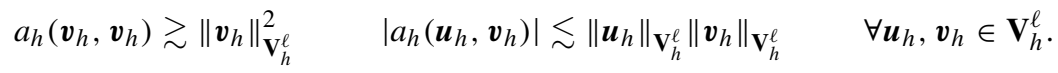

The coercivity bound is achieved provided that the penalty parameter $\gamma_{v}$ in Definition 1 is chosen sufficiently large.

\subsection{Generalized inf-sup Condition}

In this section, we prove a generalized inf-sup condition for the discrete bilinear form $b_{h}(\cdot, \cdot)$ defined in (4c). First, we need some preliminary results.

Lemma 5 (Boundedness of $\Pi^{\ell}$ in the energy norm (9)) Let Assumption 3.1 be valid. Then, we have

$$
\left\|\Pi^{\ell} \boldsymbol{v}\right\|_{\mathbf{V}_{h}^{\ell}} \lesssim \ell^{1 / 2}\|\boldsymbol{v}\|_{H^{1}(\Omega)} \quad \forall \boldsymbol{v} \in \boldsymbol{V} .
$$

The proof follows using Lemma 3, Assumption A.2, and the continuity of the Stein operator $\mathcal{E}$ in (10). Further, we introduce the $L^{2}$ projection operator

$$
\Pi_{0}^{\ell}:\left[L^{2}(\Omega)\right]^{d} \longrightarrow \mathbf{V}_{h}^{\ell}, \quad\left(\boldsymbol{w}_{h}, \boldsymbol{v}-\Pi_{0}^{\ell} \boldsymbol{v}\right)_{L^{2}(\Omega)}=0 \quad \forall \boldsymbol{w}_{h} \in \mathbf{V}_{h}^{\ell}
$$

We state a stability result for the projector $\Pi_{0}^{\ell}$, which is based on a further assumption and a technical result; see Assumption 3.2 and Lemma 6 below, respectively.

Assumption 3.2 The decomposition $\left\{\mathcal{T}_{h}\right\}_{h}$ is shape-regular. Moreover, for all $h>0$, each element $K$ of $\mathcal{T}_{h}$ is $p$-coverable, i.e., there exist $m_{K} \in \mathbb{N}$ overlapping shape-regular simplices $K_{i}, i=1, \ldots, m_{K}$, such that

$$
\operatorname{dist}\left(K, \partial K_{i}\right)<C_{a s} \frac{\operatorname{diam}\left(K_{i}\right)}{p^{2}} \quad \text { and } \quad\left|K_{i}\right| \geq c_{a s}|K|,
$$

where $c_{a s}$ and $C_{a s}$ are positive constants independent of $\mathcal{T}_{h}$ and $K$.

The following inverse estimate on shape-regular polygons can be found, e.g., in [27, Lemma 14]. It generalizes a similar result for standard geometries; see, e.g., [55, Theorem 4.76] and is based on Assumption 3.2. Such an assumption can be weakened; see Remark 2 below.

Lemma 6 ( $H^{1}-L^{2}$ polynomial inverse estimate) Let Assumption 3.2 be valid. For each $K \in \mathcal{T}_{h}$, the following polynomial inverse inequality is valid:

$$
\|\nabla v\|_{L^{2}(K)}^{2} \lesssim \frac{\ell^{4}}{h_{K}^{2}}\|v\|_{L^{2}(K)}^{2} \quad \forall v \in \mathcal{P}^{\ell}(K), \quad \ell \geq 1 .
$$

Based on employing the above result, we prove the following bound.

Lemma 7 (Stability properties of orthogonal projections) Let Assumptions 3.1 and 3.2 be valid. Then, we have

$$
\left\|\Pi_{0}^{\ell}\left(\boldsymbol{v}-\Pi^{\ell} \boldsymbol{v}\right)\right\|_{\mathbf{V}_{h}^{\ell}} \lesssim \ell\|\boldsymbol{v}\|_{\boldsymbol{V}} \quad \forall \boldsymbol{v} \in \boldsymbol{V}
$$


From the definition of norm (9), the proof follows using the inverse estimate in Lemma 6 , the stability of the projector $\Pi_{0}^{\ell}$, the polynomial approximation results in Lemma 2, and the continuity of the Stein operator $\mathcal{E}$ in (10).

Remark 1 Assumption 3.2 is required in the proof of the polynomial inverse estimate of Lemma 6 . On the other hand, the suboptimality in terms of the polynomial degree in the stability properties detailed in Lemma 7 is now due to both the inverse estimates of Lemmata 1 and 6. This propagates further in the proof of the discrete inf-sup condition, see Proposition 1 below, and consequently to the abstract convergence analysis detailed in Sect. 5 below.

Remark 2 Following the recent approach of [25], it is possible to prove the inverse estimates in Lemmata 1 and 6 using assumptions milder than Assumptions 3.1 and 3.2. Notably, the theory therein presented covers very general geometries, including $\mathcal{C}^{1}$-curved faces and possibly the presence of arbitrary number of faces.

Next, we show that a generalized inf-sup condition is valid, provided that the polynomial degrees $\ell$ and $m$ of the discrete velocity and pressure spaces satisfy $m-\ell \leq 1$. This condition guarantees in fact that $\nabla Q_{h}^{m} \subseteq \mathbf{V}_{h}^{\ell}$.

Proposition 1 (Generalized inf-sup condition for $b_{h}(\cdot, \cdot)$ ) Let Assumptions 3.1 and 3.2 be valid and assume that the polynomial degrees $\ell$ and $m$ of the discrete velocity and pressure spaces satisfy $m-\ell \leq 1$. Then,

$$
\sup _{\mathbf{0} \neq \boldsymbol{v}_{h} \in \mathbf{V}_{h}^{\ell}} \frac{b_{h}\left(q_{h}, \boldsymbol{v}_{h}\right)}{\left\|\boldsymbol{v}_{h}\right\|_{\mathbf{V}_{h}^{\ell}}}+\left|q_{h}\right|_{\jmath} \geq \beta_{h}\left\|q_{h}\right\|_{L^{2}(\Omega)} \quad \forall q_{h} \in Q_{h}^{m},
$$

where the discrete inf-sup constant behaves as

$$
\beta_{h}=O\left(\frac{\beta}{\max \left\{\ell^{1 / 2}\left(1+\ell^{1 / 2}\right), m^{1 / 2}+1\right\}}\right) .
$$

Proof We provide the main steps of the proof. Upon employing element-wise integration by parts, the bilinear form $b_{h}(\cdot, \cdot)$ can be rewritten as

$$
b_{h}\left(q_{h}, \boldsymbol{v}_{h}\right)=\int_{\Omega} \nabla_{h} q_{h} \cdot \boldsymbol{v}_{h}-\sum_{F \in \mathcal{F}_{h}^{i}} \int_{F} \llbracket q_{h} \rrbracket \cdot\left\{\boldsymbol{v}_{h}\right\} \quad \forall q_{h} \in Q_{h}^{m}, \boldsymbol{v}_{h} \in \mathbf{V}_{h}^{\ell} .
$$

Recall the continuous inf-sup condition (3): there exists $\beta>0$ such that, to each $q_{h} \in Q_{h}^{m} \subset$ $L_{0}^{2}(\Omega)$, we associate $\boldsymbol{v}_{q_{h}} \in \boldsymbol{V}$ with

$$
\nabla \cdot \boldsymbol{v}_{q_{h}}=q_{h}, \quad \beta\left\|\boldsymbol{v}_{q_{h}}\right\|_{\boldsymbol{V}} \leq\left\|q_{h}\right\|_{L^{2}(\Omega)} .
$$

Then, applying element-wise integration by parts, using that $\llbracket \boldsymbol{v}_{q_{h}} \rrbracket=\mathbf{0}$ for any $F \in \mathcal{F}_{h}$, and observing that $\nabla_{h} q_{h} \in \mathbf{V}_{h}^{\ell}$ if $\ell \geq m-1$, we obtain

$$
\begin{aligned}
& \left\|q_{h}\right\|_{L^{2}(\Omega)}^{2}=\int_{\Omega} q_{h} \nabla \cdot \boldsymbol{v}_{q_{h}}=-\int_{\Omega} \nabla_{h} q_{h} \cdot \boldsymbol{v}_{q_{h}}+\sum_{F \in \mathcal{F}_{h}} \int_{F} \llbracket q_{h} \rrbracket:\left\{\boldsymbol{v}_{q_{h}}\right\} \\
& =\underbrace{-b_{h}\left(q_{h}, \Pi^{\ell} \boldsymbol{v}_{q_{h}}\right)}+\underbrace{\int_{\Omega} \nabla_{h} q_{h} \cdot\left(\Pi^{\ell} \boldsymbol{v}_{q_{h}}-\boldsymbol{v}_{q_{h}}\right)}+\underbrace{\sum_{F \in \mathcal{F}_{h}} \int_{F} \llbracket q_{h} \rrbracket \cdot\left\{\boldsymbol{v}_{q_{h}}-\Pi^{\ell} \boldsymbol{v}_{q_{h}}\right\}} .
\end{aligned}
$$


We bound the three terms on the right-hand side separately. As for the term (A), thanks to Lemma 5 and (12), we get

$$
\text { A } \leq \frac{\left|b_{h}\left(q_{h}, \Pi^{\ell} \boldsymbol{v}_{q_{h}}\right)\right|}{\left\|\Pi^{\ell} \boldsymbol{v}_{q_{h}}\right\|_{\mathbf{V}_{h}^{\ell}}}\left\|\Pi^{\ell} \boldsymbol{v}_{q_{h}}\right\|_{\mathbf{V}_{h}^{\ell}} \lesssim \frac{\ell^{1 / 2}}{\beta}\left\|q_{h}\right\|_{L^{2}(\Omega)} \sup _{\boldsymbol{v}_{h} \in \mathbf{V}_{h}^{\ell} \backslash\{\boldsymbol{0}\}} \frac{b_{h}\left(q_{h}, \boldsymbol{v}_{h}\right)}{\left\|\boldsymbol{v}_{h}\right\|_{\mathbf{V}_{h}^{\ell}}} .
$$

As for the term (C), using the Cauchy-Schwarz inequality, the definition of $\sigma_{p}$ in Definition 1 , Assumption A.3, the continuous trace inequality and Lemma 2 with $r=1$, and $m-\ell \leq 1$, we obtain

$$
\mathrm{C} \lesssim\left|q_{h}\right|_{\jmath}\left(\sum_{K \in \mathcal{T}_{h}} \frac{m}{h_{K}}\left\|\boldsymbol{v}_{q_{h}}-\Pi^{\ell} \boldsymbol{v}_{q_{h}}\right\|_{L^{2}(\partial K)}^{2}\right)^{1 / 2} \lesssim \frac{1}{\beta}\left|q_{h}\right|_{\jmath}\left\|q_{h}\right\|_{L^{2}(\Omega)},
$$

where we have also used the continuity of the Stein extension operator $\mathcal{E}$ in (10), Assumption A.3, and the continuous inf-sup condition (12). As for the term B), using the definition of $L^{2}$ projector, the fact that $\nabla_{h} q_{h} \in \mathbf{V}_{h}^{\ell}$, an integration by parts, and, proceeding as before, we write

$$
\text { (B) } \begin{aligned}
& \left.=b_{h}\left(q_{h}, \Pi_{0}^{\ell}\left(\Pi^{\ell} \boldsymbol{v}_{q_{h}}-\boldsymbol{v}_{q_{h}}\right)\right)+\sum_{F \in \mathcal{F}_{h}^{i}} \int_{F} \llbracket q_{h} \rrbracket \cdot\left\{\Pi_{0}^{\ell}\left(\Pi^{\ell} \boldsymbol{v}_{q_{h}}-\boldsymbol{v}_{q_{h}}\right)\right)\right\} \\
& \lesssim \frac{\ell}{\beta}\left(\sup _{\boldsymbol{v}_{h} \in \mathbf{V}_{h}^{\ell} \backslash\{\boldsymbol{0}\}} \frac{b_{h}\left(q_{h}, \boldsymbol{v}_{h}\right)}{\left\|\boldsymbol{v}_{h}\right\|_{\mathbf{V}_{h}^{\ell}}}\right)\left\|q_{h}\right\|_{L^{2}(\Omega)}+\frac{m^{1 / 2}}{\beta}\left|q_{h}\right|_{\jmath}\left\|q_{h}\right\|_{L^{2}(\Omega)} .
\end{aligned}
$$

Collecting the previous estimates, we arrive at

$$
\left\|q_{h}\right\|_{L^{2}(\Omega)} \lesssim \frac{1}{\beta} \max \left\{\ell^{1 / 2}\left(1+\ell^{1 / 2}\right), m^{1 / 2}+1\right\}\left(\sup _{\boldsymbol{v}_{h} \in \mathbf{V}_{h}^{\ell} \backslash\{\mathbf{0}\}} \frac{b_{h}\left(q_{h}, \boldsymbol{v}_{h}\right)}{\left\|\boldsymbol{v}_{h}\right\|_{\mathbf{V}_{h}^{\ell}}}+\left|q_{h}\right|_{\jmath}\right) .
$$

The assertion follows with $\beta_{h}$ as in (11).

Remark 3 The constant of the generalized inf-sup condition stated in Proposition 1 is uniform with respect to the mesh size but depends on the polynomial approximation degrees $\ell$ and $m$; see (11). This implies that $\beta_{h} \searrow 0$ as $\ell, m \nearrow+\infty$. In Sect. 4, we shall present some computations to assess numerically the sharpness of the inf-sup constant $\beta_{h}$, for different mesh configurations and polynomial orders. We will find out that Assumption 3.2 does not seem necessary in the proof of Proposition 1; see Remark 2. The analysis with milder assumptions is under investigation.

\subsection{Well-Posedness of the Discrete Stokes Problem via the Banach-Necas-Babuška Theorem}

In this section, we show that (7) is well-posed. To this aim, we first notice that

$$
\begin{aligned}
\mathcal{B}_{h}\left(\left(\boldsymbol{u}_{h}, p_{h}\right) ;\left(\boldsymbol{u}_{h}, p_{h}\right)\right) & =a_{h}\left(\boldsymbol{u}_{h}, \boldsymbol{u}_{h}\right)+b_{h}\left(p_{h}, \boldsymbol{u}_{h}\right)-b_{h}\left(p_{h}, \boldsymbol{u}_{h}\right)+s_{h}\left(p_{h}, p_{h}\right) \\
& \gtrsim\left\|\boldsymbol{u}_{h}\right\|_{\mathbf{V}_{h}^{\ell}}^{2}+\left|p_{h}\right|_{\jmath}^{2} \quad \forall\left(\boldsymbol{u}_{h}, p_{h}\right) \in \mathbf{V}_{h}^{\ell} \times Q_{h}^{m},
\end{aligned}
$$

provided that Assumption 3.1 is valid and the stabilization constant $\gamma_{v}$ in Definition 1 is chosen sufficiently large. The following result implies the well-posedness of the discrete problem (7), 
based on employing the Banach-Nečas-Babuška theorem; see [36, Theorem 2.6] and [37, Theorem 25.9], cf. also e.g, [32, Theorem 1.1 and Sect. 6.1.2.3].

Lemma 8 Under the hypotheses of Proposition 1 and assuming that the stabilization constant $\gamma_{v}$ appearing in Definition 1 is chosen sufficiently large, there hold:

1. there exists $\alpha>0$ such that

$$
\inf _{\left(\boldsymbol{u}_{h}, p_{h}\right) \in \mathbf{V}_{h}^{\ell} \times Q_{h}^{m}} \sup _{\left(\boldsymbol{v}_{h}, q_{h}\right) \in \mathbf{V}_{h}^{\ell} \times Q_{h}^{m}} \frac{\mathcal{B}_{h}\left(\left(\boldsymbol{u}_{h}, p_{h}\right) ;\left(\boldsymbol{v}_{h}, q_{h}\right)\right)}{\left\|\left(\boldsymbol{u}_{h}, p_{h}\right)\right\|_{\mathrm{E}}\left\|\left(\boldsymbol{v}_{h}, q_{h}\right)\right\|_{\mathrm{E}}} \geq \alpha ;
$$

2. for any $\left(\boldsymbol{u}_{h}, p_{h}\right) \in \mathbf{V}_{h}^{\ell} \times Q_{h}^{m}$ such that

$$
\mathcal{B}_{h}\left(\left(\boldsymbol{u}_{h}, p_{h}\right),\left(\boldsymbol{v}_{h}, q_{h}\right)\right)=0 \text { for all }\left(\boldsymbol{v}_{h}, q_{h}\right) \in \mathbf{V}_{h}^{\ell} \times Q_{h}^{m},
$$

[BNB(ii)]

then $\left(\boldsymbol{u}_{h}, p_{h}\right)=(\mathbf{0}, 0)$.

Proof We first show [BNB(i)]. Given $\left(\boldsymbol{u}_{h}, p_{h}\right) \in \mathbf{V}_{h}^{\ell} \times Q_{h}^{m}$, we have

$$
\mathcal{B}_{h}\left(\left(\boldsymbol{u}_{h}, p_{h}\right) ;\left(\boldsymbol{u}_{h}, p_{h}\right)\right) \leq \mathbb{M}\left\|\left(\boldsymbol{u}_{h}, p_{h}\right)\right\|_{\mathrm{E}},
$$

where $\|\cdot\|_{\mathrm{E}}$ is defined as in (8) and

$$
\mathbb{M}=\sup _{\substack{\left(\boldsymbol{v}_{h}, q_{h}\right) \in \mathbf{V}_{h}^{\ell} \times Q_{h}^{m} \\\left(\boldsymbol{v}_{h}, q_{h}\right) \neq(\mathbf{0}, 0)}} \frac{\mathcal{B}_{h}\left(\left(\boldsymbol{u}_{h}, p_{h}\right) ;\left(\boldsymbol{v}_{h}, q_{h}\right)\right)}{\left\|\left(\boldsymbol{v}_{h}, q_{h}\right)\right\|_{\mathrm{E}}} .
$$

Using (13) and (14), we get

$$
\left\|\boldsymbol{u}_{h}\right\|_{\mathbf{V}_{h}^{\ell}}^{2}+\left|p_{h}\right|_{\jmath}^{2} \lesssim \mathcal{B}_{h}\left(\left(\boldsymbol{u}_{h}, p_{h}\right) ;\left(\boldsymbol{u}_{h}, p_{h}\right)\right) \leq \mathbb{M}\left\|\left(\boldsymbol{u}_{h}, p_{h}\right)\right\|_{\mathrm{E}} .
$$

Thanks to Proposition 1 and the fact that

$$
b_{h}\left(p_{h}, \boldsymbol{v}_{h}\right)=\mathcal{B}_{h}\left(\left(\boldsymbol{u}_{h}, p_{h}\right),\left(\boldsymbol{v}_{h}, 0\right)\right)-a_{h}\left(\boldsymbol{u}_{h}, \boldsymbol{v}_{h}\right) \quad \forall \boldsymbol{v}_{h} \in \mathbf{V}_{h}^{\ell},
$$

we have

$$
\begin{aligned}
\beta_{h}\left\|p_{h}\right\|_{L^{2}(\Omega)} & \leq \sup _{\mathbf{0} \neq \boldsymbol{v}_{h} \in \mathbf{V}_{h}^{\ell}} \frac{b_{h}\left(p_{h}, \boldsymbol{v}_{h}\right)}{\left\|\boldsymbol{v}_{h}\right\|_{\mathbf{V}_{h}^{\ell}}}+\left|p_{h}\right|_{\jmath} \\
& =\sup _{\mathbf{0} \neq \boldsymbol{v}_{h} \in \mathbf{V}_{h}^{\ell}} \frac{\mathcal{B}_{h}\left(\left(\boldsymbol{u}_{h}, p_{h}\right),\left(\boldsymbol{v}_{h}, 0\right)\right)-a_{h}\left(\boldsymbol{u}_{h}, \boldsymbol{v}_{h}\right)}{\left\|\boldsymbol{v}_{h}\right\|_{\mathbf{V}_{h}^{\ell}}}+\left|p_{h}\right|_{\jmath} \\
& \leq \sup _{\mathbf{0} \neq \boldsymbol{v}_{h} \in \mathbf{V}_{h}^{\ell}} \frac{\left|\mathcal{B}_{h}\left(\left(\boldsymbol{u}_{h}, p_{h}\right),\left(\boldsymbol{v}_{h}, 0\right)\right)\right|}{\left\|\left(\boldsymbol{v}_{h}, 0\right)\right\|_{\mathrm{E}}}+\sup _{\mathbf{0 \neq} \neq \boldsymbol{v}_{h} \in \mathbf{V}_{h}^{\ell}} \frac{\left|a_{h}\left(\boldsymbol{u}_{h}, \boldsymbol{v}_{h}\right)\right|}{\left\|\boldsymbol{v}_{h}\right\|_{\mathbf{V}_{h}^{\ell}}}+\left|p_{h}\right|_{\jmath} \\
& \lesssim \sup _{\mathbf{0} \neq \boldsymbol{v}_{h} \in \mathbf{V}_{h}^{\ell}} \frac{\left|\mathcal{B}_{h}\left(\left(\boldsymbol{u}_{h}, p_{h}\right),\left(\boldsymbol{v}_{h}, 0\right)\right)\right|}{\left\|\left(\boldsymbol{v}_{h}, 0\right)\right\|_{\mathrm{E}}}+\left\|\boldsymbol{u}_{h}\right\|_{\mathbf{V}_{h}^{\ell}}+\left|p_{h}\right|_{\jmath} \\
& \leq \mathbb{M}+\left\|\boldsymbol{u}_{h}\right\|_{\mathbf{V}_{h}^{\ell}}+\left|p_{h}\right|_{\jmath} .
\end{aligned}
$$

Using (15), we deduce

$$
\beta_{h}^{2}\left\|p_{h}\right\|_{L^{2}(\Omega)}^{2} \lesssim \mathbb{M}^{2}+\left\|\boldsymbol{u}_{h}\right\|_{\mathbf{V}_{h}^{\ell}}^{2}+\left|p_{h}\right|_{\jmath}^{2} \leq \mathbb{M}^{2}+\mathbb{M}\left\|\left(\boldsymbol{u}_{h}, p_{h}\right)\right\|_{\mathrm{E}} .
$$


Using the definition of $\|(\cdot, \cdot)\|_{\mathrm{E}},(15)$, the above bound, and a Young's inequality with a positive parameter $\gamma$, we have

$$
\begin{aligned}
& \beta_{h}^{2}\left\|\left(\boldsymbol{u}_{h}, p_{h}\right)\right\|_{\mathrm{E}}^{2}=\beta_{h}^{2}\left(\left\|\boldsymbol{u}_{h}\right\|_{\mathbf{V}_{h}^{\ell}}^{2}+\left\|p_{h}\right\|_{L^{2}(\Omega)}^{2}+\left|p_{h}\right|_{\jmath}^{2}\right) \\
& \quad \lesssim \beta_{h}^{2} \mathbb{M}\left\|\left(\boldsymbol{u}_{h}, p_{h}\right)\right\|_{\mathrm{E}}+\beta_{h}^{2}\left\|p_{h}\right\|_{L^{2}(\Omega)}^{2} \lesssim \beta_{h}^{2} \mathbb{M}\left\|\left(\boldsymbol{u}_{h}, p_{h}\right)\right\|_{\mathrm{E}}+\mathbb{M}^{2}+\mathbb{M}\left\|\left(\boldsymbol{u}_{h}, p_{h}\right)\right\|_{\mathrm{E}} \\
& \quad=\left(1+\beta_{h}^{2}\right) \mathbb{M}\left\|\left(\boldsymbol{u}_{h}, p_{h}\right)\right\|_{\mathrm{E}}+\mathbb{M}^{2} \leq \gamma\left\|\left(\boldsymbol{u}_{h}, p_{h}\right)\right\|_{\mathrm{E}}^{2}+\left(1+\frac{\left(1+\beta_{h}^{2}\right)^{2}}{\gamma}\right) \mathbb{M}^{2} .
\end{aligned}
$$

Thus, we write

$$
\left(\beta_{h}^{2}-\gamma\right)\left\|\left(\boldsymbol{u}_{h}, p_{h}\right)\right\|_{\mathrm{E}}^{2} \lesssim\left(1+\frac{\left(1+\beta_{h}^{2}\right)^{2}}{\gamma}\right) \mathbb{M}^{2} .
$$

Choosing $\gamma$ to be equal to $\beta_{h}^{2} / 2$, we arrive at

$$
\left\|\left(\boldsymbol{u}_{h}, p_{h}\right)\right\|_{\mathrm{E}}^{2} \lesssim\left(\frac{1}{\beta_{h}^{2}}+\frac{\left(1+\beta_{h}^{2}\right)^{2}}{\beta_{h}^{4}}\right) \mathbb{M}^{2} \lesssim \frac{1}{\beta_{h}^{4}} \mathbb{M}^{2},
$$

i.e., $\left\|\left(\boldsymbol{u}_{h}, p_{h}\right)\right\|_{\mathrm{E}} \lesssim \alpha \mathbb{M}$ with $\alpha=O\left(\beta_{h}^{-2}\right)$. The assertion follows from the definition of $\mathbb{M}$.

Next, we observe that, provided that the stabilization constant $\gamma_{v}$ appearing in Definition 1 is chosen sufficiently large and since we are in finite-dimensional setting with trial and test spaces having the same dimension, [BNB(ii)] is a consequence of $[\mathrm{BNB}(\mathrm{i})]$, and the proof is complete.

Summarizing, we eventually state the main result of the section, namely the well-posedness of problem (7).

Theorem 1 Under the hypotheses of Proposition 1 and assuming that the stabilization constant $\gamma_{v}$ appearing in Definition 1 is chosen sufficiently large, [BNB(i)] and [BNB(ii)] of Lemma 8 are valid. The constant $\alpha$ in ([BNB(i)]) satisfies $\alpha=O\left(\beta_{h}^{-2}\right)$, where $\beta_{h}$ is defined as in Proposition 1. Therefore, thanks to Banach-Nečas-Babuška theorem, the discrete problem (7) is well-posed.

Remark 4 The constant $\alpha$ in ([BNB(i)]) deteriorates as the polynomial degree grows. This is due to the use of the polynomial inverse estimates, which yields a discrete inPf-sup constant depending on the polynomial degree.

\section{Numerical Evaluation of the Generalized inf-sup Constant}

Denote the shape functions of $\boldsymbol{V}_{h}^{\ell}$ and $Q_{h}^{m}$ by $\left\{\boldsymbol{\varphi}_{i}\right\}_{i=1}^{N_{u}}$ and $\left\{\psi_{j}\right\}_{j=1}^{N_{p}}$, and the corresponding number of degrees of freedom by $N_{\boldsymbol{u}}$ and $N_{p}$, respectively. We write $\boldsymbol{u}_{h}=\sum_{j=1}^{N_{\boldsymbol{u}}} u_{j} \boldsymbol{\varphi}_{i}$ and $p_{h}=\sum_{j=1}^{N_{p}} p_{j} \psi_{i}$. The algebraic form of the stationary Stokes problem corresponding to the problem in (5) reads

$$
\left[\begin{array}{cc}
A_{h} & B_{h}^{T} \\
B_{h} & -S_{h}
\end{array}\right]\left[\begin{array}{l}
\boldsymbol{U} \\
\boldsymbol{P}
\end{array}\right]=\left[\begin{array}{c}
\boldsymbol{F}_{\boldsymbol{h}} \\
\mathbf{0}
\end{array}\right],
$$

where $\boldsymbol{U} \in \mathbb{R}^{N_{u}}$ and $\boldsymbol{P} \in \mathbb{R}^{N_{p}}$ are the vectors collecting the expansion coefficients $\left\{u_{j}\right\}_{j}$ and $\left\{p_{j}\right\}_{j}$, respectively, whereas $A_{h}, B_{h}, S_{h}$, and $f_{\boldsymbol{h}}$ denote the matrix representations of 
the discrete bilinear forms in (4b)-(4d) and right-hand side in (5). We recall that the discrete inf-sup condition given in Proposition 1 is given by

$$
\sup _{\mathbf{0} \neq \boldsymbol{v}_{h} \in \mathbf{V}_{h}^{\ell}} \frac{b_{h}\left(q_{h}, \boldsymbol{v}_{h}\right)}{\left\|\boldsymbol{v}_{h}\right\|_{\mathbf{V}_{h}^{\ell}}}+\eta\left|q_{h}\right|_{\jmath} \geq \beta_{h}\left\|q_{h}\right\|_{L^{2}(\Omega)} \quad \forall q_{h} \in Q_{h}^{m} .
$$

Here, we have added a parameter $\eta=\{0,1\}$ to address numerically the case where no pressure stabilization is added in the discrete formulation. Introduce the generalized eigenvalue problem

$$
G_{h} x=\lambda T_{h} x,
$$

with $G_{h}=B_{h} A_{h}^{-1} B_{h}^{T}+\eta S_{h}, T_{h}=M_{h}$, where $M_{h}$ is the mass matrix. We distinguish two cases:

- If $\eta=1$ (pressure stabilization), the discrete inf-sup constant satisfies

$$
\beta_{h}\left\langle M_{h} \boldsymbol{q}, \boldsymbol{q}\right\rangle^{1 / 2} \leq\left\langle B_{h} A_{h}^{-1} B_{h}^{T} \boldsymbol{q}, \boldsymbol{q}\right\rangle^{1 / 2}+\left\langle S_{h} \boldsymbol{q}, \boldsymbol{q}\right\rangle^{1 / 2} \quad \forall \boldsymbol{q} \in \mathbb{R}^{N_{p}}, \boldsymbol{q} \neq \mathbf{1} .
$$

By noting that $a+b \leq(\sqrt{a}+\sqrt{b})^{2} \leq 2 a+2 b$, we have

$$
\begin{aligned}
& \beta_{h}^{2}\left\langle M_{h} \boldsymbol{q}, \boldsymbol{q}\right\rangle \leq 2\left\langle B_{h} A_{h}^{-1} B_{h}^{T} \boldsymbol{q}, \boldsymbol{q}\right\rangle+2\left\langle S_{h} \boldsymbol{q}, \boldsymbol{q}\right\rangle \\
& =2\left\langle\left(B_{h} A_{h}^{-1} B_{h}^{T}+S_{h}\right) \boldsymbol{q}, \boldsymbol{q}\right\rangle \\
& \quad \forall \boldsymbol{q} \in \mathbb{R}^{N_{p}}, \boldsymbol{q} \neq 1
\end{aligned}
$$

and hence

$$
\beta_{h}^{2}=\min _{\substack{\boldsymbol{q} \in \mathbb{R}^{N_{p}} \\ \boldsymbol{q} \neq \mathbf{1}}} \frac{2\left\langle\left(B_{h} A_{h}^{-1} B_{h}^{T}+S_{h}\right) \boldsymbol{q}, \boldsymbol{q}\right\rangle}{\left\langle M_{h} \boldsymbol{q}, \boldsymbol{q}\right\rangle}=\min _{\substack{\boldsymbol{q} \in \mathbb{R}^{N_{p}} \\ \boldsymbol{q} \neq \mathbf{1}}} \frac{2\left\langle G_{h} \boldsymbol{q}, \boldsymbol{q}\right\rangle}{\left\langle T_{h} \boldsymbol{q}, \boldsymbol{q}\right\rangle} .
$$

The discrete inf-sup condition we used above is only valid in $Q_{h}^{m}$, which means for pressures $q_{h}$ with zero mean value. This allows us to exclude $\boldsymbol{q}=\mathbf{1}$ in the minimum above. Moreover, introduce

$$
F(\boldsymbol{q}):=\frac{\left\langle G_{h} \boldsymbol{q}, \boldsymbol{q}\right\rangle}{\left\langle T_{h} \boldsymbol{q}, \boldsymbol{q}\right\rangle} .
$$

By linearity, we have $F(k \mathbf{1})=F(\mathbf{1})$. This allows us also to exclude $\boldsymbol{q}=k \mathbf{1}$ in the minimum above as well.

- If $\eta=0$ (no pressure stabilization), the discrete inf-sup constant satisfies

$$
\begin{aligned}
\beta_{h} & =\min _{\substack{\boldsymbol{q} \in \mathbb{R}^{N_{p}} \\
\boldsymbol{q} \neq \mathbf{1}}} \max _{\substack{\boldsymbol{v} \in \mathbb{R}^{N_{u}} \\
\boldsymbol{v} \neq \mathbf{0}}} \frac{\left|\left\langle\boldsymbol{q}, B_{h} \boldsymbol{v}\right\rangle\right|}{\left\langle A_{h} \boldsymbol{v}, \boldsymbol{v}\right\rangle^{1 / 2}\left\langle M_{h} \boldsymbol{q}, \boldsymbol{q}\right\rangle^{1 / 2}} \\
& =\min _{\substack{\boldsymbol{q} \in \mathbb{R}^{N_{p}} \\
\boldsymbol{q} \neq \mathbf{1}}} \frac{1}{\left\langle M_{h} \boldsymbol{q}, \boldsymbol{q}\right\rangle^{1 / 2}} \max _{\substack{\boldsymbol{w} \in \mathbb{R}^{N_{\boldsymbol{u}}} \\
\boldsymbol{w}=A_{h}^{1 / 2} \boldsymbol{v} \neq \mathbf{0}}} \frac{\left|\left\langle\boldsymbol{q}, B_{h} A_{h}^{-1 / 2} \boldsymbol{w}\right\rangle\right|}{\langle\boldsymbol{w}, \boldsymbol{w}\rangle^{1 / 2}} \\
& =\min _{\substack{\boldsymbol{q} \in \mathbb{R}^{N_{p}} \\
\boldsymbol{q} \neq \mathbf{1}}} \frac{1}{\left\langle M_{h} \boldsymbol{q}, \boldsymbol{q}\right\rangle^{1 / 2}} \max _{\substack{\boldsymbol{w} \in \mathbb{R}^{N_{\boldsymbol{u}}} \\
\boldsymbol{w} \neq \mathbf{0}}} \frac{\left|\left\langle A_{h}^{-1 / 2} B_{h}^{T} \boldsymbol{q}, \boldsymbol{w}\right\rangle\right|}{\langle\boldsymbol{w}, \boldsymbol{w}\rangle^{1 / 2}} .
\end{aligned}
$$



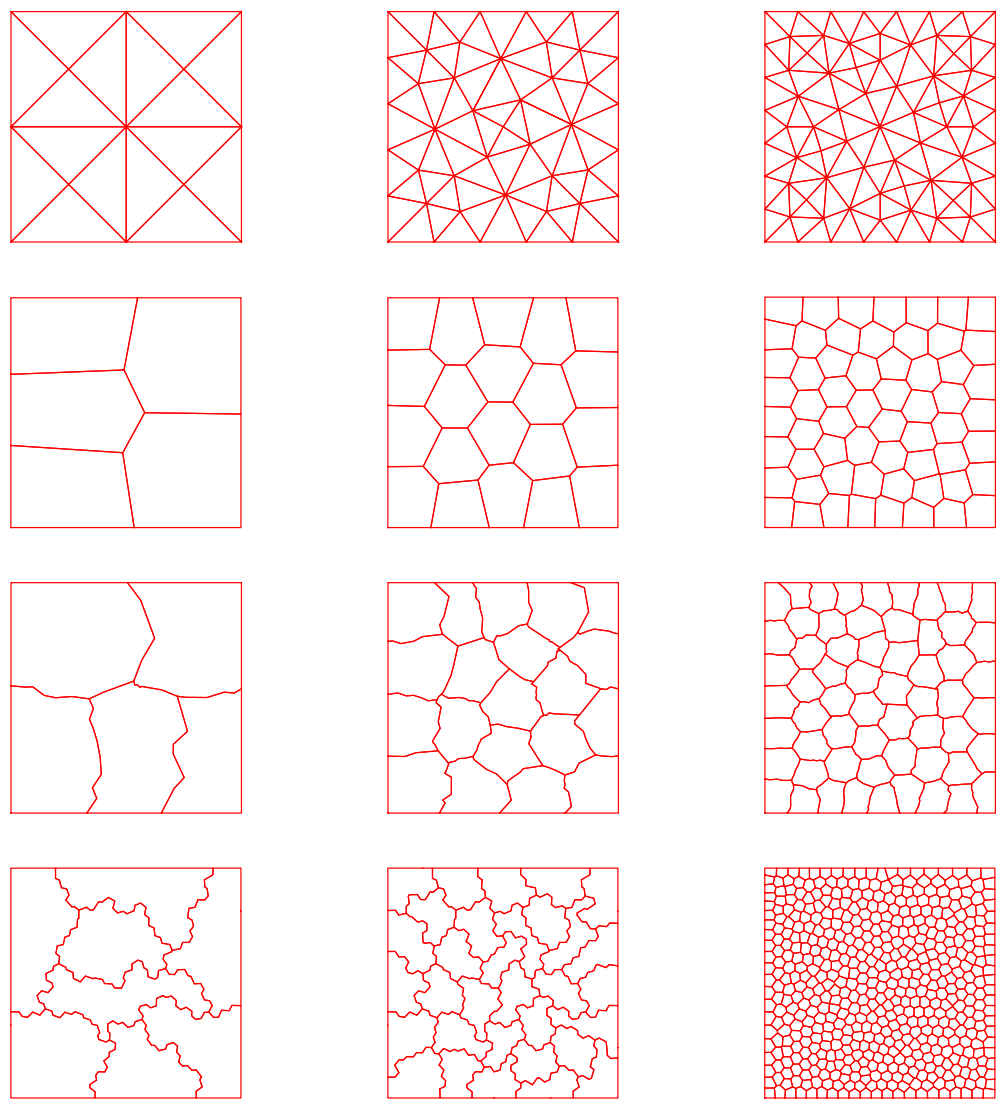

Fig. 1 From top to bottom: triangular meshes with $N_{e l}=16,68,124$ (from left to right); regular polygonal meshes with $N_{e l}=5,20,60$ (from left to right); distorted polygonal meshes with $N_{e l}=5,20,60$ (from left to right); agglomerated polygonal meshes with $N_{e l}=8,32,512$ (from left to right)

By noting that the maximum is realized for $\boldsymbol{w}=A_{h}^{-1 / 2} B_{h}^{T} \boldsymbol{q}$, we have

$$
\begin{aligned}
& \beta_{h}^{2}=\min _{\substack{\boldsymbol{q} \in \mathbb{R}^{N_{p}} \\
\boldsymbol{q} \neq \mathbf{1}}} \frac{\left\langle A_{h}^{-1 / 2} B_{h}^{T} \boldsymbol{q}, A_{h}^{-1 / 2} B_{h}^{T} \boldsymbol{q}\right\rangle}{\left\langle M_{h} \boldsymbol{q}, \boldsymbol{q}\right\rangle}=\min _{\substack{\boldsymbol{q} \in \mathbb{R}^{N_{p}} \\
\boldsymbol{q} \neq \mathbf{1}}} \frac{\left\langle B_{h} A_{h}^{-1} B_{h}^{T} \boldsymbol{q}, \boldsymbol{q}\right\rangle}{\left\langle M_{h} \boldsymbol{q}, \boldsymbol{q}\right\rangle} \\
& =\min _{\substack{\boldsymbol{q} \in \mathbb{R}^{N p} \\
\boldsymbol{q} \neq \mathbf{1}}} \frac{\left\langle G_{h} \boldsymbol{q}, \boldsymbol{q}\right\rangle}{\left\langle T_{h} \boldsymbol{q}, \boldsymbol{q}\right\rangle} .
\end{aligned}
$$

By solving the discrete eigenvalue problem in Eq. (16), we have that $\beta_{h} \approx \min _{\lambda_{i}>0} \sqrt{\lambda_{i}}$. To estimate numerically $\beta_{h}$, we consider the Stokes problem on the unit square domain $\Omega=(0,1)^{2}$. We computed $\beta_{h}$ on several sequences of meshes, namely triangular, regular, distorted, and agglomerated polygonal meshes; see Fig. 1 for an illustrative example of the considered grids. The regular polygonal meshes have been generated via PolyMesher [58], while the distorted polygonal ones are generated starting from a regular grid and randomly adding grid nodes on the edges to obtain elements with a large number of possibly degenerating edges. The resulting elements may be non-convex. The sequence of agglomerated 
polygonal meshes are generated by agglomerating elements starting from an initial Voronoi tessellation; see the last row of Fig. 1. To solve the generalized eigenvalue problem (16), we employ the eigs command of Matlab.

We first investigate the behaviour of $\beta_{h}$ for fixed polynomial approximation orders for the velocity and the pressure and varying the mesh size. In Figs. 2 and 3, we report the computed values of $\beta_{h}$ as a function of the mesh size $h$ for different mesh configurations and different choices of the discrete velocity and pressure spaces $\mathcal{P}^{m+k}-\mathcal{P}^{m}, k=0,1,2,3,4$. In the stabilized cases $\eta=1$, the constant $\beta_{h}$ is uniformly bounded from 0 independently of the mesh size. This is in agreement with the result shown in Proposition 1. Furthermore, as predicted in Proposition 1, $\beta_{h}$ depends on $m$ for all the considered mesh configurations, at least when $m=\ell$. From the numerical computations obtained in the no pressure stabilization cases $\eta=0$ with $k=1,2,3$, 4, we draw the following conclusions: ( $i) \beta_{h}$ is independent of $h$ for all the considered mesh configurations except for agglomerated meshes, where we can detect a mild dependence; (ii) the dependence of $\beta_{h}$ on the velocity and pressure polynomial approximation degrees is stronger than in the stabilized case.

Next, we investigate the behaviour of $\beta_{h}$ by varying the polynomial approximation orders for the velocity and the pressure spaces, and fixing the computational mesh. In Fig. 4, we report the computed value of $\beta_{h}$ as a function of the polynomial approximation degree $m$ for different choices of the velocity and pressure spaces $\mathcal{P}^{m+k}-\mathcal{P}^{m}, k=0,1,2,3,4$. We set the parameter $\eta=1$ for $k=0,1,2$, and $\eta=0$ for $k=1,2,3,4$. When $\eta=1$, we obtain the following results. For $k=0$, i.e., $\ell=m$, the dependence of $\beta_{h}$ is in agreement with Proposition 1: the constant $\beta_{h}$ deteriorates as $m$ grows. Nevertheless, the estimate in Proposition 1 is slightly suboptimal by a factor of $m^{-1 / 2}$, as our numerical computations suggest that $\beta_{h}=O\left(m^{-1 / 2}\right)$ for all the considered mesh configurations. For $k=1,2$, on triangular and regular polygonal meshes, $\beta_{h}$ looks independent of $m$, while it mildly depends on $m$ on irregular and agglomerated polygonal meshes. From the numerical computations obtained in the no pressure stabilization cases $\eta=0$, we draw the following conclusions: $(i)$ on regular polygonal meshes, $\beta_{h}$ is independent of $m$ for all the considered velocity-pressure pairs $\mathcal{P}^{m+k}-\mathcal{P}^{m}, k=1,2,3,4$; (ii) on irregular and agglomerated polygonal meshes, the behaviour of $\beta_{h}$ is less clear and we detect a mild dependence on $m$. By comparing the cases $\mathcal{P}^{m+k}-\mathcal{P}^{m}, k=1,2$ with and without pressure stabilization, at least for the agglomerated polygonal meshes, the dependence of $\beta_{h}$ on $m$ is milder for the case $\eta=1$.

Moreover, we study the behaviour of $\beta_{h}$ in the case of degenerate edges, i.e., when the number of the edges of a polygon with fixed size increases and the size of the edges tends to zero. In particular, we consider an initial triangular mesh with a uniform mesh size; see Fig. 5(left). Starting from this grid, we generate a sequence of meshes by halving recursively the edges of the element at the center of the mesh, leading to a polygon with an increasing number of edges; see Fig. 5(middle and right). We indicate the number of the edges of the polygon with \#edges. In Fig. 6, we report the values of $\beta_{h}$ as a function of \#edges for different choices of the discrete velocity and pressure spaces $\mathcal{P}^{m+k}-\mathcal{P}^{m}, k=0,1,2$, with $(\eta=1)$ and without $(\eta=0)$ pressure stabilization. The computed numerical inf-sup constant $\beta_{h}$ seems to be independent of the size of the edges for any choice of $k$ and the pressure stabilization term. This indicates that Assumption 3.2 in Proposition 1 may be relaxed; see Remark 3.

Finally, we consider a sequence of grids that mimics fluid meshes typically appearing in fluid-structure interaction applications; see Sect. 7 below. These grids are generated as follows: first, consider a uniform regular triangular mesh of a square domain; next, carve the domain out and get a hole inside it. For example, this hole may represent a structure domain immersed in a fluid one. In the proximity of the hole, the resulting mesh presents polygonal elements that may be non-convex, of arbitrary size and of anisotropic shape. We 

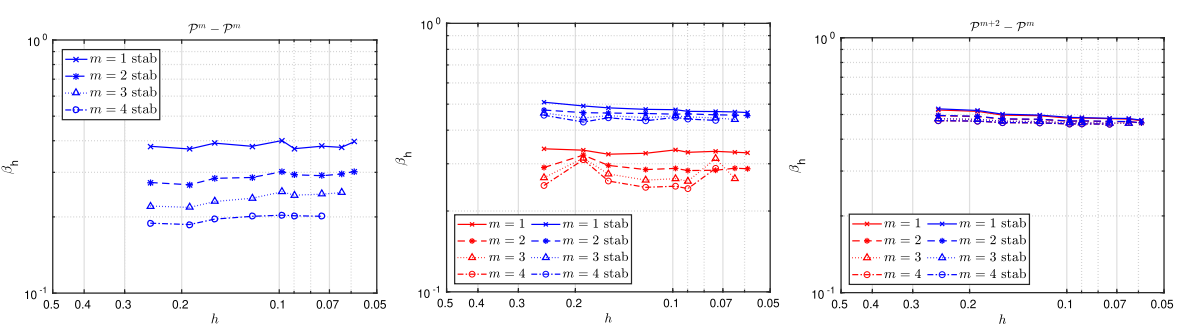

(a) Triangular grids
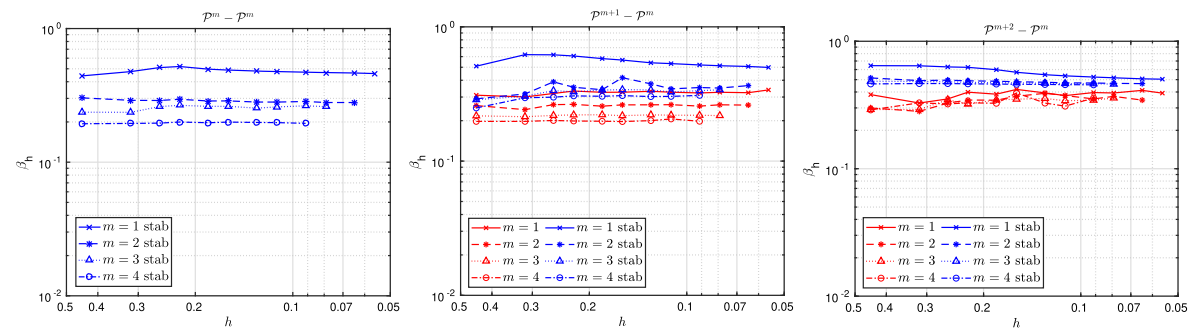

(b) Regular polygonal meshes
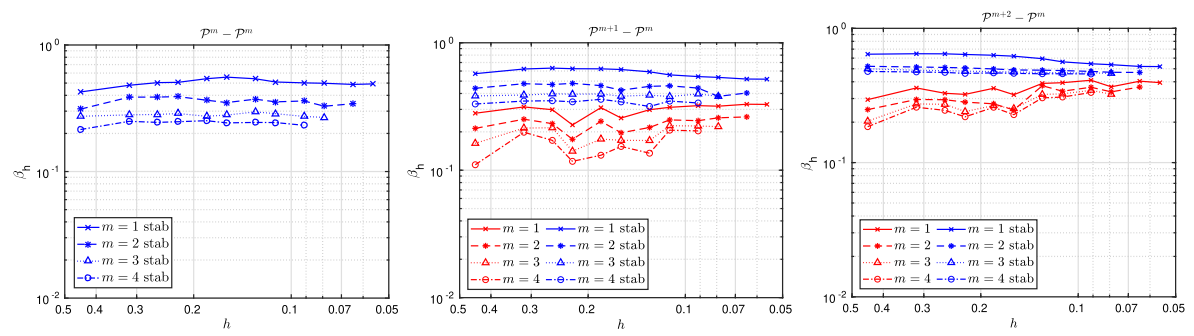

(c) Distorted polygonal meshes
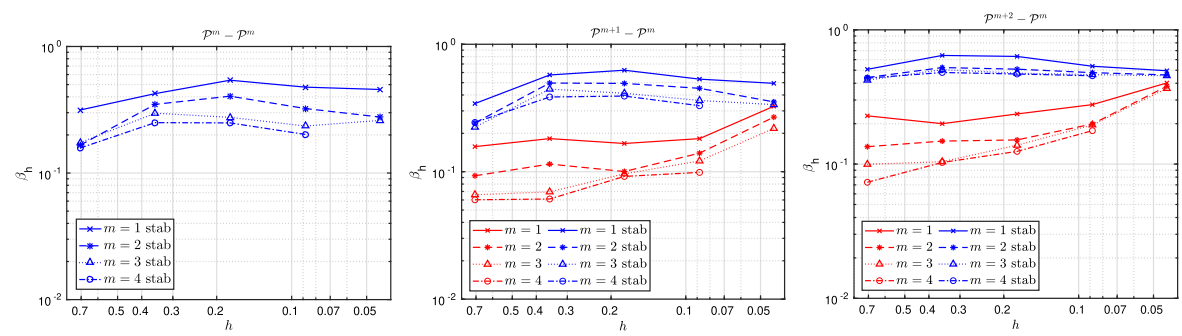

(d) Agglomerated polygonal meshes

Fig. 2 Values of $\beta_{h}$ as function of the mesh size $h$ for different choices of the polynomial degree for the discrete velocity and pressure spaces $\mathcal{P}^{m+k}-\mathcal{P}^{m}$, computed solving the generalized eigenvalue problem (16). From left to right: $\mathcal{P}^{m}-\mathcal{P}^{m}, \mathcal{P}^{m+1}-\mathcal{P}^{m}, \mathcal{P}^{m+2}-\mathcal{P}^{m}$. The parameter $\eta=1$ for $k=0,1,2$ (blue lines), and $\eta=0$ for $k=1,2$ (red lines) (Color figure online)

consider a slender rectangular hole placed in the center of the square domain that rotates around its center of mass, see Fig. 7, and we study the behaviour of the discrete inf-sup constant $\beta_{h}$ by varying the angle of rotation $\theta$ of the hole. In Fig. 8, we plot the value of the discrete inf-sup constant as a function of the angle $\theta$, by spanning the range $\left[0^{\circ}, 180^{\circ}\right]$ with step $1^{\circ}$, for different choices of the discrete velocity and pressure spaces $\mathcal{P}^{m+k}-\mathcal{P}^{m}$, 

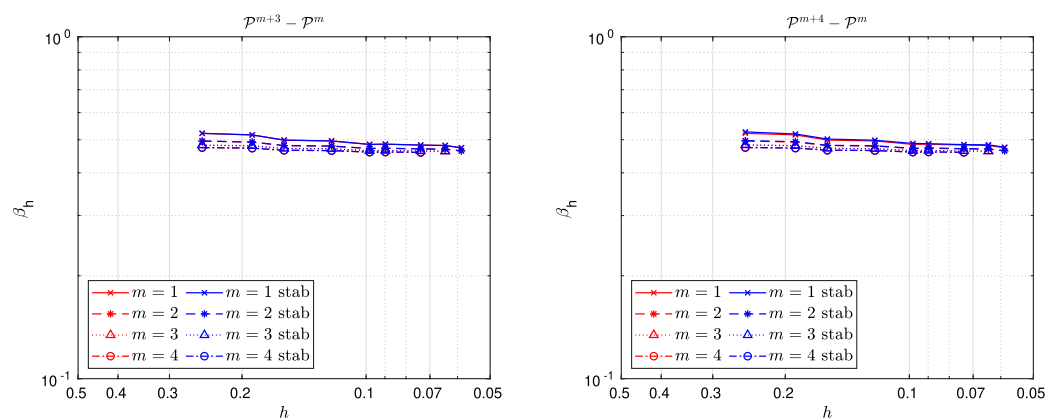

(a) Triangular grids
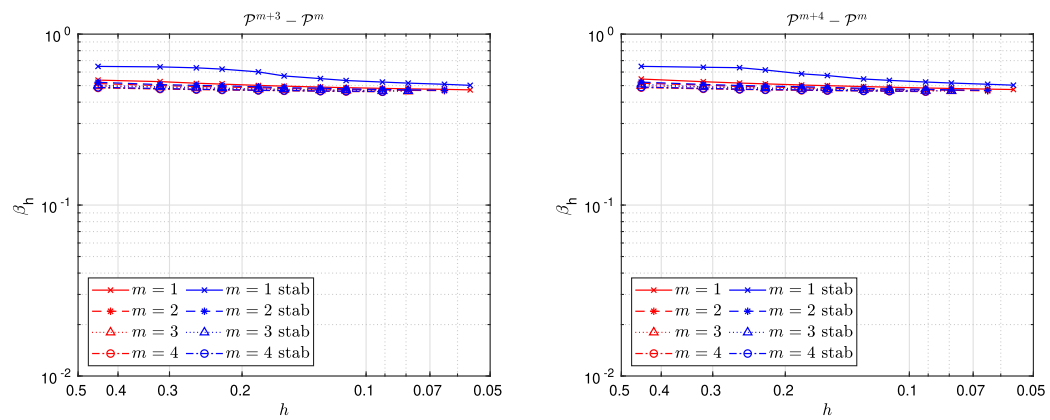

(b) Regular polygonal meshes
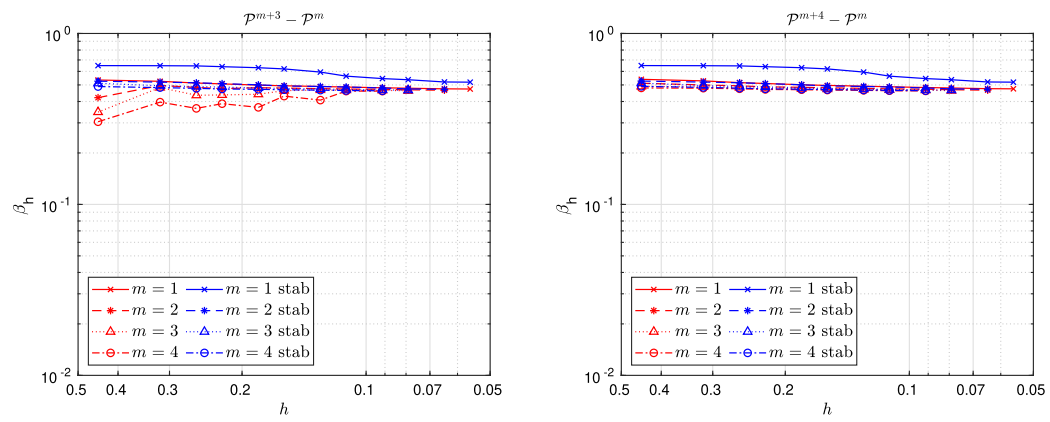

(c) Distorted polygonal meshes
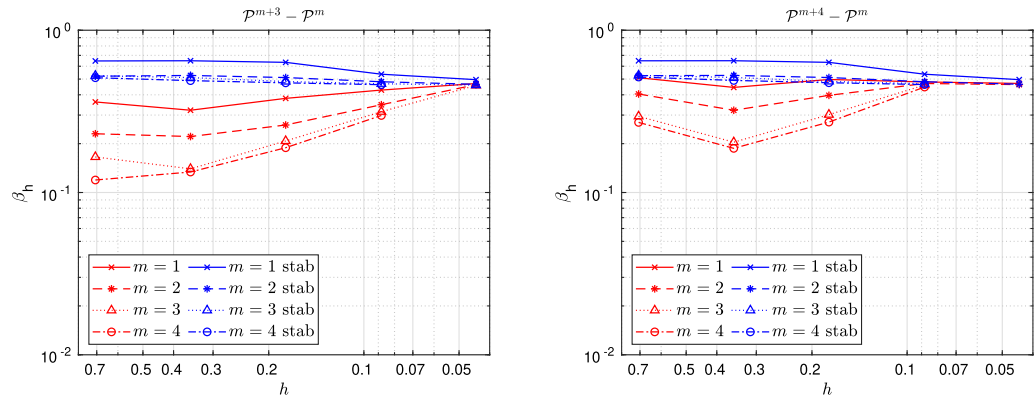

(d) Agglomerated polygonal meshes

Fig. 3 Values of $\beta_{h}$ as function of the mesh size $h$ for different choices of the polynomial degree for the discrete velocity and pressure spaces $\mathcal{P}^{m+k}-\mathcal{P}^{m}$, computed solving the generalized eigenvalue problem (16). From left to right: $\mathcal{P}^{m+3}-\mathcal{P}^{m}, \mathcal{P}^{m+4}-\mathcal{P}^{m}$. The parameter $\eta=1$ (blue lines) and $\eta=0$ (red lines) (Color figure online) 


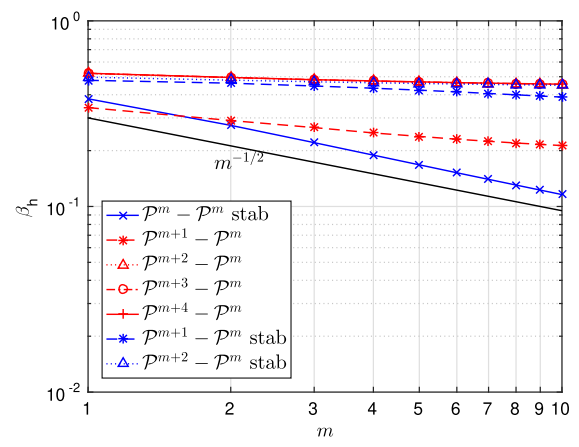

(a) Triangular grids $\left(N_{e l}=16\right)$

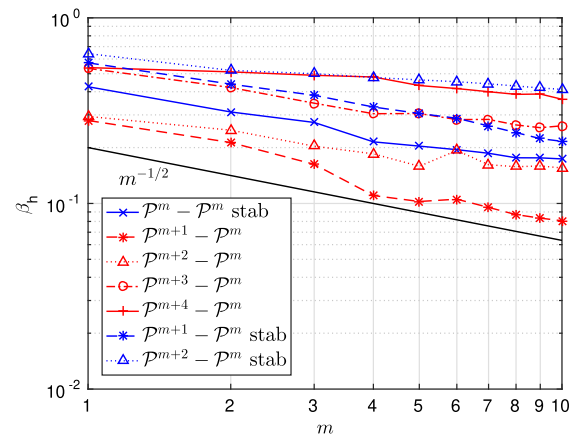

(c) Distorted polygonal meshes $\left(N_{e l}=5\right)$

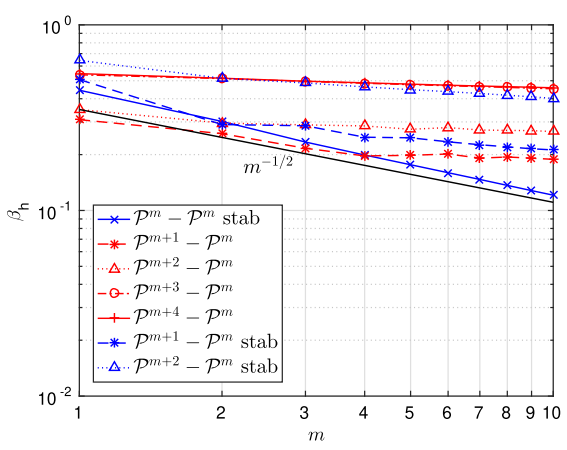

(b) Regular polygonal meshes $\left(N_{e l}=5\right)$

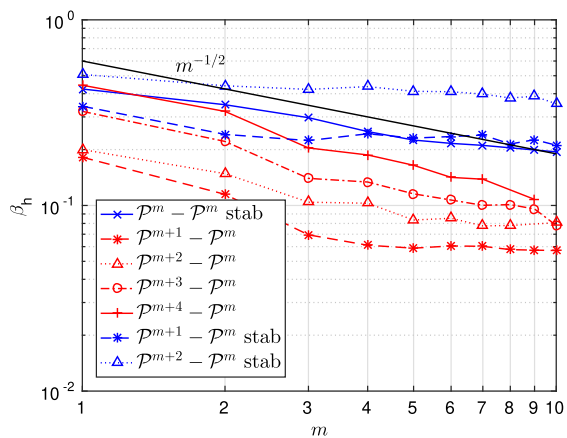

(d) Agglomerated polygonal meshes $\left(N_{e l}=8\right)$

Fig. 4 Values of $\beta_{h}$ as function of the polynomial degree for different choices of the velocity and pressure spaces $\mathcal{P}^{m+k}-\mathcal{P}^{m}, k=0,1,2,3,4$ computed solving the generalized eigenvalue problem (16). The parameter $\eta=1$ if $k=0,1,2$ (blue lines), and $\eta=0$ if $k=1,2,3,4$ (red lines) (Color figure online)
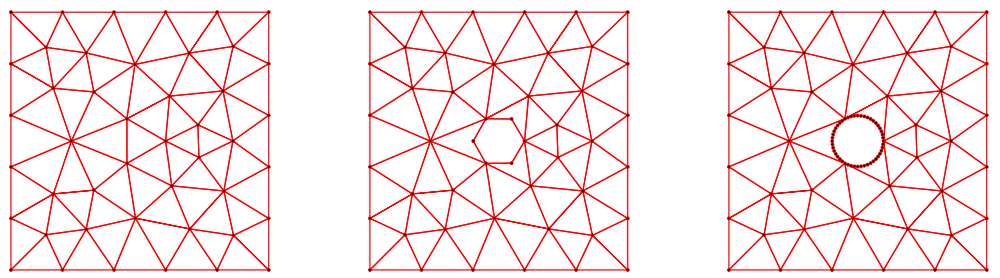

Fig. 5 Meshes obtained with a recursive splitting of the edges of the element at the center. From left to right: initial triangular meshes with \#edges $=3$; mesh at the first iteration with \#edges =6; mesh at the fourth iteration with \#edges $=48$

$k=0,1,2, m=1,2$, with and without the pressure stabilization term. The presence of small or anisotropic elements only slightly deteriorates the constant $\beta_{h}$ for the nonstabilized case $(\eta=0)$, while they seem irrelevant for the stabilized case $(\eta=1)$.

\section{A Priori Error Estimates for the Stationary Stokes Problem}

We introduce the spaces

$$
\mathcal{X}=\mathbf{V}_{h}^{\ell}+\left(\left[H^{2}(\Omega)\right]^{d} \cap\left[H_{0}^{1}(\Omega)\right]^{d}\right), \quad \mathcal{M}=Q_{h}^{m}+\left(H^{1}(\Omega) \cap L_{0}^{2}(\Omega)\right)
$$



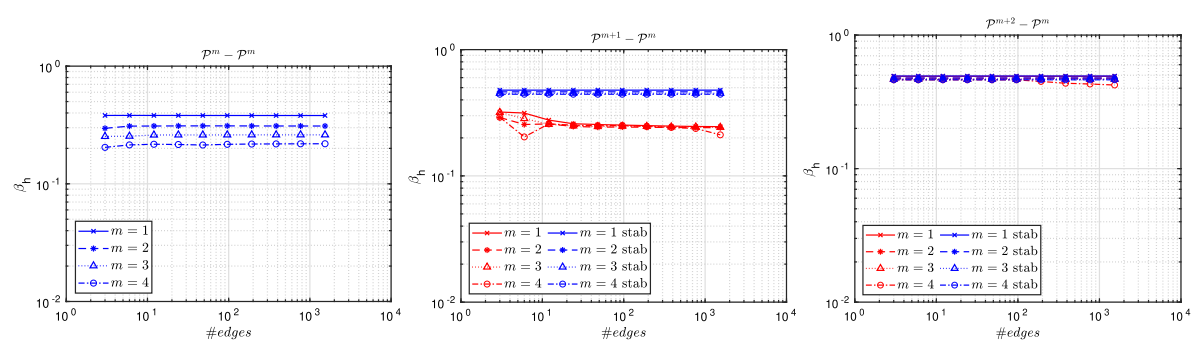

Fig. 6 Values of $\beta_{h}$ as function of the number of edges \#edges for different choices of the polynomial degree for the discrete velocity and pressure spaces $\mathcal{P}^{m+k}-\mathcal{P}^{m}$, computed solving the generalized eigenvalue problem (16). From left to right: $\mathcal{P}^{m}-\mathcal{P}^{m}, \mathcal{P}^{m+1}-\mathcal{P}^{m}, \mathcal{P}^{m+2}-\mathcal{P}^{m}$. The parameter $\eta=1$ for $k=0,1,2$ (blue lines), and $\eta=0$ for $k=1,2$ (red lines) (Color figure online)
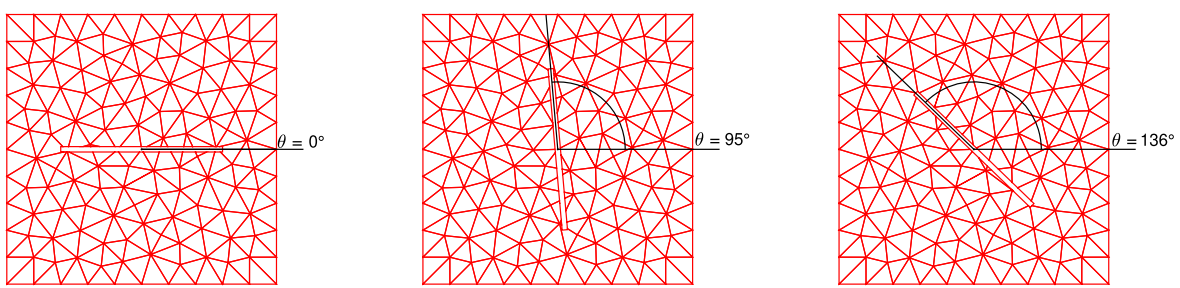

Fig. 7 Polygonal meshes obtained by rotating the hole placed in the center of the initial triangular mesh. Small or anisotropic elements appear. From left to right: polygonal mesh obtained for $\theta=0^{\circ}$; polygonal mesh obtained for $\theta=95^{\circ}$; polygonal mesh obtained for $\theta=136^{\circ}$
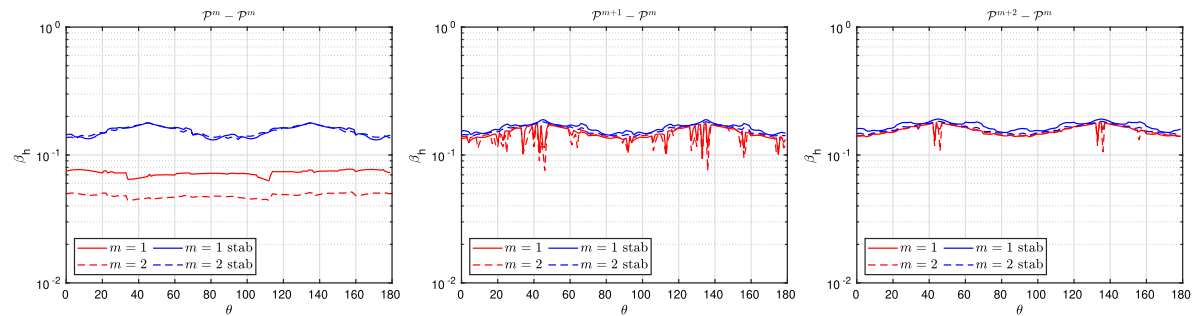

Fig. 8 Values of $\beta_{h}$ as function of the angle of rotation $\theta$ for different choices of the polynomial degree for the discrete velocity and pressure spaces $\mathcal{P}^{m+k}-\mathcal{P}^{m}$, computed solving the generalized eigenvalue problem (16). From left to right: $\mathcal{P}^{m}-\mathcal{P}^{m}, \mathcal{P}^{m+1}-\mathcal{P}^{m}, \mathcal{P}^{m+2}-\mathcal{P}^{m}$. The parameter $\eta=1$ (blue lines) and $\eta=0$ (red lines) (Color figure online)

for the velocity and pressure, respectively. For all $(\boldsymbol{u}, p),(\boldsymbol{v}, q) \in \mathcal{X} \times \mathcal{M}$, we consider the extension of the discrete bilinear form $\mathcal{B}_{h}$ introduced in equation (6) to $\mathcal{X} \times \mathcal{M}$. We endow $\mathcal{X} \times \mathcal{M}$ with the energy norm

$$
\|(\boldsymbol{v}, q)\|_{\mathcal{X} \times \mathcal{M}}^{2}=\|(\boldsymbol{v}, q)\|_{\mathrm{E}}^{2}+\sum_{F \in \mathcal{F}_{h}}\left\|\sigma_{v}^{-1 / 2}\{p \boldsymbol{I}\}\right\|_{L^{2}(F)}^{2}+\sum_{F \in \mathcal{F}_{h}}\left\|\sigma_{v}^{-1 / 2}\left\{\nabla_{h} \boldsymbol{u}\right\}\right\|_{L^{2}(F)}^{2} .
$$

We now state the main result of the section. The proof follows the steps of [32, Theorem 6.21] and classical arguments of DG methods, and is omitted for the sake of brevity.

Theorem 2 (Abstract error estimate) Let $(\boldsymbol{u}, p) \in \mathcal{X} \times \mathcal{M}$ be the solution to the steady Stokes problem, i.e., (2) with $\rho=0$. Let Assumptions 3.1 and 3.2 be valid and $\left(\boldsymbol{u}_{h}, p_{h}\right) \in \mathbf{V}_{h}^{\ell} \times Q_{h}^{m}$, 
$m-\ell \leq 1$, be the solution of (7). The following error estimate is valid:

$$
\left\|\left(\boldsymbol{u}-\boldsymbol{u}_{h}, p-p_{h}\right)\right\|_{\mathcal{X} \times \mathcal{M}} \leq\left(1+\frac{C}{\beta_{h}}\right) \inf _{\left(\boldsymbol{v}_{h}, q_{h}\right) \in \mathbf{V}_{h}^{\ell} \times Q_{h}^{m}}\left\|\left(\boldsymbol{u}-\boldsymbol{v}_{h}, p-q_{h}\right)\right\|_{\mathcal{X} \times \mathcal{M}}
$$

where $C$ is the continuity constant of the bilinear form $\mathcal{B}_{h}(\cdot, \cdot)$ in the norm (17) and $\alpha=$ $O\left(\beta_{h}^{2}\right)$ is the inf-sup constant of Theorem 1 .

Finally, by employing the approximation results reported in Sect. 3 with Theorem 2, we show the $h p$-version a priori error estimates.

Corollary 1 (Convergence rate in the energy norm) Let $\mathcal{T}_{h}$ be a polytopic mesh and $\mathcal{T}_{h}^{\#}$ be the corresponding covering satisfying Definition 2, the hypotheses of Theorem 2 be valid, and $m-\ell \leq 1$. If, for any $K \in \mathcal{T}_{h},\left.(\boldsymbol{u}, p)\right|_{K} \in H^{r_{u}}(K) \times H^{r_{p}}(K)$ with $r_{u} \geq 2$ and $r_{p} \geq 1$, such that for any $\mathcal{K} \in \mathcal{T}_{h}^{\#}, K \subset \mathcal{K},\left.(\mathcal{E} \boldsymbol{u}, \mathcal{E} p)\right|_{\mathcal{K}} \in H^{r_{u}}(\mathcal{K}) \times H^{r_{p}}(\mathcal{K})$, then

$$
\left\|\left(\boldsymbol{u}-\boldsymbol{u}_{h}, p-p_{h}\right)\right\|_{\mathcal{X} \times \mathcal{M}}^{2} \lesssim \frac{1}{\beta_{h}} \sum_{K \in \mathcal{T}_{h}}\left(\frac{h_{K}^{2\left(s_{u}-1\right)}}{\ell^{2\left(r_{u}-3 / 2\right)}}\|\mathcal{E} \boldsymbol{u}\|_{H^{r_{u}(\mathcal{K})}}^{2}+\frac{h_{K}^{2 s_{p}}}{m^{2 r_{p}}}\|\mathcal{E} p\|_{H^{r_{p}}(\mathcal{K})}^{2} \cdot\right)
$$

where $s_{u}=\min \left\{\ell+1, r_{u}\right\}, s_{p}=\min \left\{m+1, r_{p}\right\}$ and $\beta_{h}$ as in (11).

Proof By considering Theorem 2, we set

$$
\mathcal{I}=\inf _{\left(\boldsymbol{v}_{h}, q_{h}\right) \in \mathbf{V}_{h}^{\ell} \times Q_{h}^{m}}\left\|\left(\boldsymbol{u}-\boldsymbol{v}_{h}, p-q_{h}\right)\right\|_{\mathcal{X} \times \mathcal{M}} .
$$

We have

$$
\mathcal{I}^{2} \leq \underbrace{\sum_{K \in \mathcal{T}_{h}}\left\|\mu^{1 / 2} \nabla\left(\boldsymbol{u}-\Pi^{\ell} \boldsymbol{u}\right)\right\|_{L^{2}(K)}^{2}+\sum_{F \in \mathcal{F}_{h}}\left\|\sigma_{v}^{1 / 2} \llbracket \boldsymbol{u}-\Pi^{\ell} \boldsymbol{u} \rrbracket\right\|_{L^{2}(F)}^{2}}
$$

(A)

$$
+\underbrace{\sum_{K \in \mathcal{T}_{h}}\left\|p-\Pi^{m} p\right\|_{L^{2}(K)}^{2}+\sum_{F \in \mathcal{F}_{h}}\left\|\sigma_{p}^{1 / 2} \llbracket p-\Pi^{m} p \rrbracket\right\|_{L^{2}(F)}^{2}}
$$

$$
+\underbrace{\sum_{F \in \mathcal{F}_{h}}\left\|\sigma_{v}^{-1 / 2}\left\{\left(p-\Pi^{m} p\right) \boldsymbol{I}\right\}\right\|_{L^{2}(F)}^{2}}+\underbrace{\sum_{F \in \mathcal{F}_{h}}\left\|\sigma_{v}^{-1 / 2}\left\{\nabla_{h}\left(\boldsymbol{u}-\Pi^{\ell} \boldsymbol{u}\right)\right\}\right\|_{L^{2}(F)}^{2}},
$$

where $\Pi^{\ell}$ and $\Pi^{m}$ denote the polynomial approximant introduced in Lemma 2. By using the continuous trace-inverse inequality, Lemmas 2 and that $m-\ell \leq 1$, we can prove the following bounds:

$$
\text { (A) }+ \text { (D) } \lesssim \sum_{K \in \mathcal{T}_{h}} \frac{h_{K}^{2\left(s_{u}-1\right)}}{\ell^{2\left(r_{u}-3 / 2\right)}}\|\mathcal{E} \boldsymbol{u}\|_{H^{r_{u}(\mathcal{K})}}^{2}, \quad \text { (B) }+ \text { C } \lesssim \sum_{K \in \mathcal{T}_{h}} \frac{h_{K}^{2 s_{p}}}{m^{2 r_{p}}}\|\mathcal{E} p\|_{H^{r_{p}(\mathcal{K})}}^{2},
$$

where $s_{u}=\min \left\{\ell+1, r_{u}\right\}$ and $s_{p}=\min \left\{m+1, r_{p}\right\}$, and the assertion follows.

Remark 5 The estimate of Corollary 1 is suboptimal with respect to the polynomial order also due to the presence of the constant $\beta_{h}$, where $\beta_{h}$ is the discrete generalized inf-sup constant introduced in Proposition 1. 
Remark 6 By assuming $h \simeq h_{K}$ for any $K \in \mathcal{T}_{h}$, uniform regularity of the solution $r_{u}=r$, $r_{p}=r-1$, and $m \approx \ell$, the estimate in Corollary 1 becomes

$$
\left\|\left(\boldsymbol{u}-\boldsymbol{u}_{h}, p-p_{h}\right)\right\|_{\mathcal{X} \times \mathcal{M}} \lesssim \frac{1}{\beta_{h}} \frac{h^{s-1}}{m^{r-3 / 2}}\left(\|\mathcal{E} \boldsymbol{u}\|_{H^{r}\left(\cup_{K \in \mathcal{T}_{h}} \mathcal{K}\right)}+\|\mathcal{E} p\|_{H^{r-1}\left(\cup_{K \in \mathcal{T}_{h}} \mathcal{K}\right)}\right)
$$

where $s=\min \{m+1, r\}$ and $\beta_{h}$ as in (11).

\section{An Application: PolyDG for FSI Problems}

In this section, we introduce a continuous FSI problem and its PolyDG discretization, with the aim of further exploring the stability properties of the PolyDG discretization of the Stokes problem and their impact on the approximation of related differential problems; see Sect. 7 below.

Let $\Omega \subset \mathbb{R}^{d}$ and $\Omega_{s} \subset \mathbb{R}^{d}, d=2,3$, be two polygonal/polyhedral domains. In $\Omega$, we consider an incompressible Newtonian fluid with density $\rho$ and dynamic viscosity $\mu$, where $\boldsymbol{u}$ and $p$ are the fluid velocity and pressure, while in $\Omega_{s}$ we consider a linear elastic material with density $\rho_{s}$, Young's modulus $E$, and Poisson's ratio $v$, where $\boldsymbol{d}$ is the solid displacement.

In what follows, we denote by $\Sigma$ the fluid-structure interface and by $\boldsymbol{n}$ its normal vector pointing outwards of $\Omega_{s}$. We indicate with $\partial \Omega$ and $\partial \Omega_{s}$ the outer boundary of the fluid and solid domain, respectively. The domains may change in time.

The fluid-structure interaction problem reads as follows: for any $t \in(0, T]$, with $T>0$, find the fluid velocity $\boldsymbol{u}=\boldsymbol{u}(t)$, the fluid pressure $p=p(t)$, and the solid displacement $\boldsymbol{d}=\boldsymbol{d}(t)$, such that

$$
\begin{array}{ll}
\rho \partial_{t} \boldsymbol{u}-\nabla \cdot \boldsymbol{T}_{f}(\boldsymbol{u}, p)=\boldsymbol{f} & \text { in } \Omega(t), \\
\nabla \cdot \boldsymbol{u}=0 & \text { in } \Omega(t), \\
\boldsymbol{u}=0 & \text { on } \partial \Omega, \\
\boldsymbol{u}=\partial_{t} \boldsymbol{d} & \text { on } \Sigma(t), \\
\boldsymbol{T}_{f}(\boldsymbol{u}, p) \boldsymbol{n}=\boldsymbol{T}_{s}(\boldsymbol{d}) \boldsymbol{n} & \text { on } \Sigma(t), \\
\rho_{s} \partial_{t t} \widehat{\boldsymbol{d}}-\nabla \cdot \widehat{\boldsymbol{T}}_{s}(\boldsymbol{d})=\boldsymbol{f}_{s} & \text { in } \widehat{\Omega}_{s}, \\
\widehat{\boldsymbol{d}}=\mathbf{0} & \text { on } \partial \widehat{\Omega}_{s},
\end{array}
$$

where $\boldsymbol{T}_{f}(\boldsymbol{u}, p)=2 \mu \boldsymbol{D}(\boldsymbol{u})-p \boldsymbol{I}$ is the fluid Cauchy stress tensor and $\widehat{\boldsymbol{T}}_{s}(\boldsymbol{d})=2 \mu_{s} \boldsymbol{D}(\widehat{\boldsymbol{d}})+$ $\lambda_{s} \nabla \cdot \widehat{\boldsymbol{d}} \boldsymbol{I}$ is the solid first Piola-Kirchhoff stress tensor, with $\boldsymbol{D}(\boldsymbol{w})=1 / 2\left(\nabla \boldsymbol{w}+\nabla^{T} \boldsymbol{w}\right)$ and $\lambda_{s}=\frac{E v}{(1+v)(1-2 v)}, \mu_{s}=\frac{E}{2(1+v)}$ are the Lamé parameters.

The structure problem is written in the reference configuration $\widehat{\Omega}_{s}=\Omega_{s}(t=0)$, and all the quantities related to the reference configuration are indicated with the $\widehat{r}$ notation.

Given the time discretization parameter $\Delta t>0$, we indicate with $t^{n}=n \Delta t, n \geq 0$, the $n$-th time step and indicate the approximation of the unknown $u$ at time $t^{n}$ by $u^{n}$. We introduce the fluid and solid meshes $\mathcal{T}_{f, h}^{n}$ and $\mathcal{T}_{s, h}^{n}$ of the fluid and solid domains $\Omega\left(t^{n}\right)$ and $\Omega_{s}\left(t^{n}\right)$, respectively. We denote the $(d-1)$-dimensional faces at time $t^{n}$ of the fluid and solid meshes by $\mathcal{F}_{f, h}^{n}$ and $\mathcal{F}_{s, h}^{n}$, respectively, except the set of faces composing the fluidstructure interface $\Sigma$ at time $t^{n}$, which are denoted by $\mathcal{F}_{\Sigma, h}^{n}$. Finally, $\boldsymbol{V}_{h}^{\ell, n}$ and $Q_{h}^{m, n}$ are the 
fluid velocity and pressure spaces evaluated at time $t^{n}$, defined as

$$
\begin{aligned}
& \boldsymbol{V}_{h}^{\ell, n}=\left\{\boldsymbol{v} \in\left[L^{2}\left(\Omega\left(t^{n}\right)\right)\right]^{d}:\left.\boldsymbol{v}\right|_{K} \in\left[\mathcal{P}^{\ell}(K)\right]^{d} \forall K \in \mathcal{T}_{f, h}^{n}\right\}, \\
& Q_{h}^{m, n}=\left\{q \in L_{0}^{2}\left(\Omega\left(t^{n}\right)\right):\left.q\right|_{K} \in \mathcal{P}^{m}(K) \forall K \in \mathcal{T}_{f, h}^{n}\right\} .
\end{aligned}
$$

The solid displacement space $\boldsymbol{W}_{h}^{\ell}$ evaluated in the reference configuration is defined as

$$
\boldsymbol{W}_{h}^{\ell}=\left\{\boldsymbol{w} \in\left[L^{2}\left(\widehat{\Omega}_{S}\right)\right]^{d}:\left.\boldsymbol{w}\right|_{K} \in\left[\mathcal{P}^{\ell}(K)\right]^{d} \forall K \in \widehat{\mathcal{T}}_{s, h}\right\} .
$$

We have assumed that the spatial polynomial order $\ell$ is the same for both the fluid velocity and the solid displacement.

Given $r \in \mathbb{N}^{+}$, we apply a Backward Difference Formula (BDF) scheme [44] of order $r$ both for the fluid and the solid subproblems. We indicate the coefficients appearing in the approximation of the first and second order time derivatives with $\xi_{i}$ and $\zeta_{i}, i=0, \ldots, r$, respectively.

Define

$$
\begin{aligned}
A_{f, h}^{n}\left(\boldsymbol{u}_{h}^{n}, p_{h}^{n} ; \boldsymbol{v}_{h}, q_{h}\right)= & \rho\left(\frac{\xi_{0}}{\Delta t} \boldsymbol{u}_{h}^{n}, \boldsymbol{v}_{h}\right)_{\Omega^{n}}+a_{f, h}^{n}\left(\boldsymbol{u}_{h}^{n}, \boldsymbol{v}_{h}\right)+b_{h}^{n}\left(p_{h}^{n}, \boldsymbol{v}_{h}\right)-b_{h}^{n}\left(q_{h}, \boldsymbol{u}_{h}^{n}\right) \\
& +s_{h}^{n}\left(p_{h}^{n}, q_{h}\right) ; \\
A_{s, h}^{n}\left(\widehat{\boldsymbol{d}}_{h}^{n}, \widehat{\boldsymbol{w}}_{h}\right)= & \rho_{s}\left(\frac{\zeta_{0}}{\Delta t^{2}} \widehat{\boldsymbol{d}}_{h}^{n}, \widehat{\boldsymbol{w}}_{h}\right)_{\widehat{\Omega}_{s}}+a_{s, h}\left(\widehat{\boldsymbol{d}}_{h}^{n}, \widehat{\boldsymbol{w}}_{h}\right) ; \\
A_{\Sigma, h}^{n}\left(\boldsymbol{u}_{h}^{n}, p_{h}^{n}, \boldsymbol{d}_{h}^{n} ; \boldsymbol{v}_{h}, q_{h}, \boldsymbol{w}_{h}\right)= & -\left(\boldsymbol{T}_{f}\left(\boldsymbol{u}_{h}^{n}, p_{h}^{n}\right) \boldsymbol{n}, \boldsymbol{v}_{h}-\boldsymbol{w}_{h}\right)_{\mathcal{F}_{\Sigma, h}^{n}} \\
& -\left(\boldsymbol{u}_{h}^{n}-\frac{\xi_{0}}{\Delta t} \boldsymbol{d}_{h}^{n}, \boldsymbol{T}_{f}\left(\boldsymbol{v}_{h},-q_{h}\right) \boldsymbol{n}\right)_{\mathcal{F}_{\Sigma, h}^{n}} \\
& +\left(\sigma_{\Sigma}\left(\boldsymbol{u}_{h}^{n}-\frac{\xi_{0}}{\Delta t} \boldsymbol{d}_{h}^{n}\right), \boldsymbol{v}_{h}-\boldsymbol{w}_{h}\right)_{\mathcal{F}_{\Sigma, h}^{n}} ; \\
F_{h}^{n}\left(\boldsymbol{v}_{h}, \boldsymbol{w}_{h}\right)= & \rho\left(\sum_{i=1}^{r} \frac{\xi_{i}}{\Delta t} \boldsymbol{u}_{h}^{n-i}, \boldsymbol{v}_{h}\right)_{\Omega^{n}}+\rho_{s}\left(\sum_{i=1}^{r} \frac{\zeta_{i}}{\Delta t^{2}} \widehat{\boldsymbol{d}}_{h}^{n-i}, \widehat{\boldsymbol{w}}_{h}\right)_{\widehat{\Omega}_{s}} \\
& +\left(\sum_{i=1}^{r} \frac{\xi_{i}}{\Delta t} \boldsymbol{d}_{h}^{n-i}, \boldsymbol{T}_{f}\left(\boldsymbol{v}_{h},-q_{h}\right) \boldsymbol{n}\right)_{\mathcal{F}_{\Sigma, h}^{n}} \\
& -\left(\sigma_{\Sigma} \sum_{i=1}^{r} \frac{\xi_{i}}{\Delta t} \boldsymbol{d}_{h}^{n-i}, \boldsymbol{v}_{h}-\boldsymbol{w}_{h}\right)_{\mathcal{F}_{\Sigma, h}^{n}}+\left(\boldsymbol{f}, \boldsymbol{v}_{h}\right)_{\Omega^{n}}+\left(\widehat{\boldsymbol{f}}_{s}, \widehat{\boldsymbol{w}}_{h}\right)_{\widehat{\Omega}_{s}} .
\end{aligned}
$$

The fully-discrete PolyDG approximation reads as follows: given $\sigma_{v} \in L^{\infty}\left(\mathcal{F}_{f, h}^{n}\right), \sigma_{p} \in$ $L^{\infty}\left(\mathcal{F}_{f, h}^{n}\right), \widehat{\sigma}_{s} \in L^{\infty}\left(\widehat{\mathcal{F}}_{s, h}\right), \sigma_{\Sigma} \in L^{\infty}\left(\mathcal{F}_{\Sigma, h}^{n}\right), \boldsymbol{f} \in\left[L^{2}\left(\Omega_{f}^{n}\right)\right]^{2}$ and $\widehat{\boldsymbol{f}}_{s} \in\left[L^{2}\left(\widehat{\Omega}_{s}\right)\right]^{2}$, for $n>0$, find $\left(\boldsymbol{u}_{h}^{n}, p_{h}^{n}, \widehat{\boldsymbol{d}}_{h}^{n}\right) \in \boldsymbol{V}_{h}^{\ell, n} \times Q_{h}^{m, n} \times \boldsymbol{W}_{h}^{\ell}$, such that

$$
A_{f, h}^{n}\left(\boldsymbol{u}_{h}^{n}, p_{h}^{n} ; \boldsymbol{v}_{h}, q_{h}\right)+A_{s, h}^{n}\left(\widehat{\boldsymbol{d}}_{h}^{n}, \widehat{\boldsymbol{w}}_{h}\right)+A_{\Sigma, h}^{n}\left(\boldsymbol{u}_{h}^{n}, p_{h}^{n}, \boldsymbol{d}_{h}^{n} ; \boldsymbol{v}_{h}, q_{h}, \boldsymbol{w}_{h}\right)=F_{h}^{n}\left(\boldsymbol{v}_{h}, \boldsymbol{w}_{h}\right),
$$

for all $\left(\boldsymbol{v}_{h}, q_{h}, \widehat{\boldsymbol{w}}_{h}\right) \in \boldsymbol{V}_{h}^{\ell, n} \times Q_{h}^{m, n} \times \boldsymbol{W}_{h}^{\ell}$.

In (18), the pressure stabilization term $s_{h}^{n}: L_{0}^{2} \times L_{0}^{2} \rightarrow \mathbb{R}$ is that given in Sect. 2.1 evaluated on $\mathcal{F}_{f, h}^{n, i}$. In (19), we have introduced the bilinear forms $a_{f, h}^{n}:\left[H^{1}\left(\mathcal{T}_{f, h}^{n}\right)\right]^{d} \times\left[H^{1}\left(\mathcal{T}_{f, h}^{n}\right)\right]^{d} \rightarrow$ 
$\mathbb{R}, b_{h}^{n}: L_{0}^{2} \times\left[H^{1}\left(\mathcal{T}_{f, h}^{n}\right)\right]^{d} \rightarrow \mathbb{R}$ and $a_{s, h}:\left[H^{1}\left(\widehat{\mathcal{T}}_{s, h}\right)\right]^{d} \times\left[H^{1}\left(\widehat{\mathcal{T}}_{s, h}\right)\right]^{d} \rightarrow \mathbb{R}$, which are defined as

$$
\begin{aligned}
a_{f, h}^{n}\left(\boldsymbol{u}_{h}^{n}, \boldsymbol{v}_{h}\right)= & \int_{\Omega}^{n} 2 \mu \boldsymbol{D}_{h}\left(\boldsymbol{u}_{h}^{n}\right): \nabla_{h} \boldsymbol{v}_{h}-\sum_{F \in \mathcal{F}_{f, h}^{n}} \int_{F} 2 \mu\left\{\boldsymbol{D}_{h}\left(\boldsymbol{u}_{h}^{n}\right)\right\}: \llbracket \boldsymbol{v}_{h} \rrbracket \\
& -\sum_{F \in \mathcal{F}_{f, h}^{n}} \int_{F} 2 \mu \llbracket \boldsymbol{u}_{h}^{n} \rrbracket:\left\{\boldsymbol{D}_{h}\left(\boldsymbol{v}_{h}\right)\right\}+\sum_{F \in \mathcal{F}_{f, h}^{n}} \int_{F} \sigma_{v} \llbracket \boldsymbol{u}_{h} \rrbracket: \llbracket \boldsymbol{v}_{h} \rrbracket, \\
b_{h}^{n}\left(p_{h}^{n}, \boldsymbol{v}_{h}^{n}\right)= & -\int_{\Omega}^{n} p_{h}^{n} \nabla_{h} \cdot \boldsymbol{v}_{h}^{n}+\sum_{F \in \mathcal{F}_{f, h}^{n}} \int_{F}\left\{p_{h}^{n} \boldsymbol{I}\right\}: \llbracket \boldsymbol{v}_{h}^{n} \rrbracket, \\
a_{s, h}\left(\widehat{\boldsymbol{d}}_{h}^{n}, \widehat{\boldsymbol{w}}_{h}\right)= & \int_{\widehat{\Omega}_{s}} 2 \mu_{s} \boldsymbol{D}_{h}\left(\widehat{\boldsymbol{d}}_{h}^{n}\right): \nabla_{h} \widehat{\boldsymbol{w}}_{h}+\int_{\widehat{\Omega}_{s}} \lambda_{s} \nabla_{h} \cdot \widehat{\boldsymbol{d}}_{h}^{n} \nabla_{h} \cdot \widehat{\boldsymbol{w}}_{h} \\
& -\sum_{F \in \widehat{\mathcal{F}}_{s, h}} \int_{F} 2 \mu_{s}\left\{\boldsymbol{D}_{h}\left(\widehat{\boldsymbol{d}}_{h}^{n}\right)\right\}: \llbracket \widehat{\boldsymbol{w}}_{h} \rrbracket-\sum_{F \in \widehat{\mathcal{F}}_{s, h}} \int_{F} \lambda_{s}\left\{\nabla_{h} \cdot \widehat{\boldsymbol{d}}_{h}^{n} \boldsymbol{I}\right\}: \llbracket \widehat{\boldsymbol{w}}_{h} \rrbracket \\
& -\sum_{F \in \widehat{\mathcal{F}}_{s, h}} \int_{F} 2 \mu_{s} \llbracket \widehat{\boldsymbol{d}}_{h}^{n} \rrbracket:\left\{\boldsymbol{D}_{h}\left(\widehat{\boldsymbol{w}}_{h}\right)\right\}-\sum_{F \in \widehat{\mathcal{F}}_{s, h}} \int_{F} \lambda_{s} \llbracket \widehat{\boldsymbol{d}}_{h}^{n} \rrbracket:\left\{\nabla_{h} \cdot \widehat{\boldsymbol{w}}_{h} \boldsymbol{I}\right\} \\
& +\sum_{F \in \widehat{\mathcal{F}}_{s, h}} \int_{F} \widehat{\sigma}_{s} \llbracket \widehat{\boldsymbol{d}}_{h}^{n} \rrbracket: \llbracket \widehat{\boldsymbol{w}}_{h} \rrbracket,
\end{aligned}
$$

where $\boldsymbol{D}_{h}(\boldsymbol{w})=1 / 2\left(\nabla_{h} \boldsymbol{w}+\nabla_{h}^{T} \boldsymbol{w}\right)$.

The functions $\sigma_{v}$ and $\sigma_{p}$ are given in Definition 1 on $\mathcal{F}_{f, h}^{n}$, while $\widehat{\sigma}_{s}: \mathcal{F}_{s, h}^{n} \rightarrow \mathbb{R}$ and $\sigma_{\Sigma}: \mathcal{F}_{\Sigma, h}^{n} \rightarrow \mathbb{R}$ are defined as

$$
\left.\widehat{\sigma}_{s}\right|_{F}=\left\{\left.\begin{array}{ll}
\gamma_{s} \max _{K^{+}, K^{-}}\left\{\frac{\ell^{2} \overline{\mathcal{C}}_{s, K}}{h_{K}}\right\} & F \in \mathcal{F}_{s, h}^{n, i}, \\
\gamma_{s} \frac{\ell^{2} \overline{\mathcal{C}}_{s, K}}{h_{K}} & F \in \mathcal{F}_{s, h}^{n, b},
\end{array} \quad \sigma_{\Sigma}\right|_{F}=\gamma_{\Sigma} \max _{K^{+}, K^{-}}\left\{\frac{\ell^{2}}{h_{K}} \mu\right\} \quad F \in \mathcal{F}_{\Sigma, h}^{n},\right.
$$

with $\gamma_{s}, \gamma_{\Sigma}$ positive constants, $\overline{\mathcal{C}}_{s, K}=\left\|\mathcal{C}_{s} \mid K\right\|_{l^{2}}$ and $\mathcal{C}_{s, K}$ the linear elasticity fourth order tensor.

Remark 7 The procedure to generate the fluid and solid meshes $\mathcal{T}_{f, h}^{n}$ and $\mathcal{T}_{s, h}^{n}$ is as follows: we first generate a background mesh $\mathcal{T}_{h}$ that covers both the fluid and solid domains and is fitted to the external boundary. Then, we generate the reference solid mesh $\widehat{\mathcal{T}}_{s, h}$ on the reference domain $\widehat{\Omega}_{s}$, so that it is fitted to the boundary $\partial \widehat{\Omega}_{s}$. These two meshes $\mathcal{T}_{h}$ and $\widehat{\mathcal{T}}_{s, h}$ are computed once and for all. Next, at the beginning of each time step, the current solid mesh $\mathcal{T}_{s, h}^{n}$ is computed by applying a transformation to $\widehat{\mathcal{T}}_{s, h}$ dictated by the numerical displacement $\widehat{\boldsymbol{d}}_{h}^{n-1}$ computed at the previous time. Finally, the current fluid mesh $\mathcal{T}_{f, h}^{n}$ is obtained by cutting off $\mathcal{T}_{s, h}^{n}$ from the background mesh $\mathcal{T}_{h}$. The resulting current fluid and solid meshes are fitted at their interface.

The integrals on the solid domain are computed in the reference configuration, i.e., on $\widehat{\mathcal{T}}_{s, h}$. The triangulation $\mathcal{T}_{s, h}^{n}$ and its corresponding set of faces $\mathcal{F}_{s, h}^{n}$ are only used to cut the mesh $\mathcal{T}_{h}$ to obtain the current fluid mesh $\mathcal{T}_{f, h}^{n}$ and to map back the solid shape functions from the current configuration to the reference one. The integrals at the fluid-structure interface are evaluated in the current configuration, by noting that $\widehat{\mathbf{d}}_{h}=\mathbf{d}_{h}^{n} \circ \mathcal{L}_{h}^{n}$, where $\mathcal{L}_{h}^{n}: \widehat{\Omega}_{s} \rightarrow \Omega_{s}^{n}$ is the discrete Lagrangian map defined as $\mathcal{L}_{h}^{n}=\mathbf{I}_{\widehat{\Omega}_{s}}+\widehat{\mathbf{d}}_{h}^{n}$. 
The fluid domain $\Omega^{n}$ and the interface $\Sigma^{n}$ in (18), (20), and (21) are unknown. Thus, they are approximated with extrapolations of order $r$ of the domains at the previous time steps, i.e., $\Omega^{n} \approx \widetilde{\Omega}^{n}=\widetilde{\Omega}^{n}\left(\Omega^{n-1}, \ldots, \Omega^{n-r}\right)$, where $\Omega^{m}=\left(\Omega \cup \Omega_{s}\right) \backslash\left(\mathcal{L}_{h}^{m}\left(\widehat{\Omega}_{s}\right)\right)$, for $m=n-1, \ldots, n-r$. In particular, the extrapolation of order $r$ of the domain $\Omega^{n}$ is obtained by using the backward finite difference formula of order $r+1$ :

$$
\Omega^{n} \approx \widetilde{\Omega}^{n}=\sum_{k=1}^{r}\left(\begin{array}{l}
r \\
k
\end{array}\right)(-1)^{k-1} \Omega^{n-k} .
$$

Due to the motion of the solid domain, $u_{h}^{n-i}$ might be undefined in parts of $\Omega^{n}$, i.e., in those cells $K \in \mathcal{T}_{h}^{n}$ that partially/fully lie outside of $\Omega^{n-i}$. In this case, i) if $K \in \mathcal{T}_{h}^{n}$ partially lies outside of $\Omega^{n-i}$, then the numerical velocity is extended based on employing an interpolation of order $\ell$; ii) if $K \in \mathcal{T}_{h}^{n}$ fully lies outside of $\Omega^{n-i}$, then an extrapolation of order $\ell$ is employed based on the value of the velocity in the neighboring fluid element; see $[8,70,71]$ and also $[3,23,48]$.

Remark 8 For the numerical stability of the FSI problem, theoretical results show that the interface terms on $\mathcal{F}_{\Sigma, h}^{n}$ of equations (20) and (21) have to be carefully defined, so that the right balance between the different contributions holds; see the pioneering [21] and more recent works [8].

\section{Numerical Results}

In this section, we present some numerical experiments for the steady Stokes problem and the time-dependent FSI problem. In Sect. 7.1, we assess the order of accuracy of the method for the steady Stokes problem as the spatial discretization parameter tends to zero and the spatial polynomial degree increases. In Sect. 7.2, we consider a FSI problem and we numerically compare the pressure field for different choices of the velocity and pressure spaces, with and without the pressure stabilization term. Finally, in Sect. 7.3, we show that the proposed PolyDG method is able to reproduce the expected dynamics of a time-dependent FSI problem. For all the proposed examples, we employ a modal expansion for the shape functions of the discrete spaces and the resulting linear systems are solved by means of a direct method.

\subsection{The Steady Stokes Problem: Convergence Results}

Here, we numerically estimate the order of convergence of the steady Stokes problem with respect to the spatial parameter $h$ when it tends to zero and the polynomial degree increases. We consider a square unit domain $\Omega=(0,1)^{2}$ and the exact solution

$$
\boldsymbol{u}_{e x}=\left[\begin{array}{c}
-\cos (2 \pi x) \sin (2 \pi y) \\
\sin (2 \pi x) \cos (2 \pi y)
\end{array}\right], \quad p_{e x}=1-e^{-x(x-1)(x-0.5)^{2}-y(y-1)(y-0.5)^{2}} .
$$

The forcing term $f$ and the Dirichlet boundary conditions are computed accordingly. We picked $\boldsymbol{u}_{e x}$ so that $\nabla \cdot \boldsymbol{u}_{e x}=0$. We set $\mu=1, \gamma_{v}=10, \gamma_{p}=10$ and $m=\ell=4$. In Fig. 9(left), we plot the error in the $L^{2}$ and $D G$-norms of the velocity, in the $L^{2}$-norm of the pressure and in the pressure semi-norm $|\cdot|_{\jmath}$ versus $h \frac{1}{\sqrt{N_{e l}}}$. The expected order of convergence are found. The error $E_{\mathrm{J}}^{p}$ seems to be superconvergent, while the error $E_{L^{2}}^{p}$ presents some slight oscillations. In Fig. 9(right), we show the errors with respect to the polynomial degree $m$, 

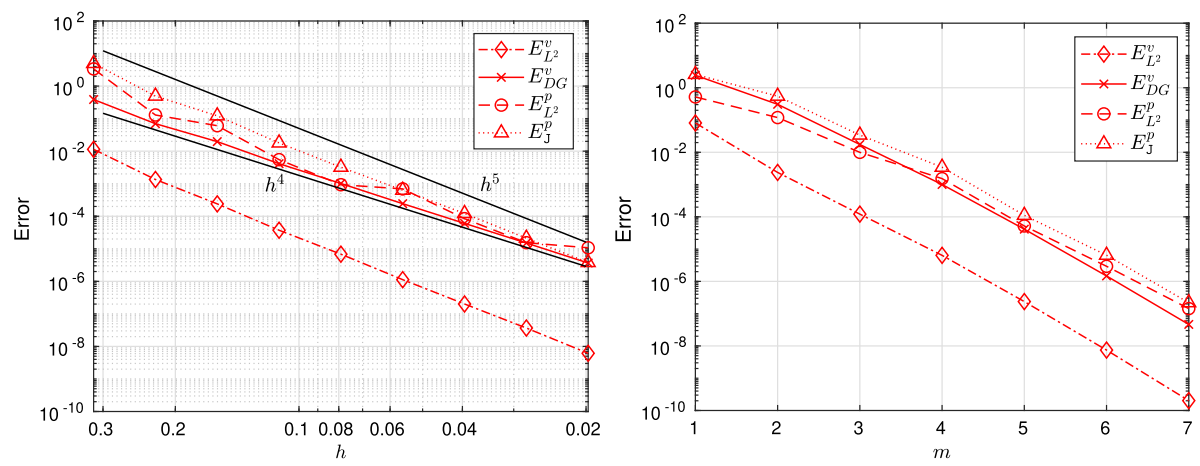

Fig. 9 Numerical estimates of the order of convergence with respect to the mesh size $h$ (left) and the polynomial degree $m$ (right)

with an underlying uniform and regular polygonal mesh, generated via PolyMesher [58], consisting of $N_{e l}=160$ elements. We observe exponential convergence in terms of the polynomial degree.

Remark 9 Within the theoretical setting of the paper, we cannot prove the exponential convergence of the $p$-version of the method. Notwithstanding, it is the expected behaviour in the standard Galerkin setting with simplicial and tensor product element meshes for analytic solutions; see, e.g., [55] and the references therein. The reason of this resides in the continuity property of the Stein extension operator (10), which is valid modulo a hidden constant depending on the involved Sobolev regularity $s$. In particular, when trying to recover exponential convergence, a term growing more than exponentially with respect to $s$ appears. A possible way to overcome this issue would be to resort to a different approach, where we assume that the solution is analytic over a slightly larger domain than $\Omega$. In particular, we should substitute the approximation result in Lemma 2 with some approximation properties by means of tensor product Legendre polynomials on tensor product element and Koornwinder polynomials on simplicial elements; see, e.g., [55] and [18] for more details, respectively. We avoid further details on this point, for it might render the understanding of the paper more cumbersome. The suboptimality in terms of the polynomial degree due to the nonrobustness of the inf-sup condition, see Remark 5, is eaten up by the expected exponential convergence for analytic solutions.

\subsection{The FSI Problem: Numerical Comparison of the Pressure Fields}

In this first numerical test we compare the pressure field for different choices of the spatial polynomial degree of the discrete velocity and pressure spaces for a FSI problem. The fluid domain $\Omega$ represents a viscous fluid with density $\rho=1 \mathrm{~g} / \mathrm{cm}^{2}$ and viscosity $\mu=0.03 \mathrm{~g} / \mathrm{s}$, while the structure domain $\Omega_{s}$ is a linear elastic barrier that horizontally divides the fluid domain in two compartments; see Fig. 10. For the structure we set the density $\rho_{s}=1.2 \mathrm{~g} / \mathrm{cm}^{2}$, the Young's modulus $E=2 \cdot 10^{4} \mathrm{dyne} / \mathrm{cm}$, and the Poisson's ratio $v=0.49$.

At the upper and lower boundaries of the fluid domain, we impose zero velocity, i.e., $\boldsymbol{u}=\mathbf{0}$, and the barrier is fixed on the left and right sides, i.e., $\boldsymbol{d}=\mathbf{0}$. To the system, initially at rest, is prescribed an inlet velocity $\boldsymbol{u}_{i n}(t)=\left(u_{i n, x}(t), 0\right) \mathrm{cm} / \mathrm{s}$ to the upper compartment 
Fig. 10 Setting of the boundary conditions on the fluid (white) and structure (grey) domains. The moving fluid-structure interface is depicted by dashed lines

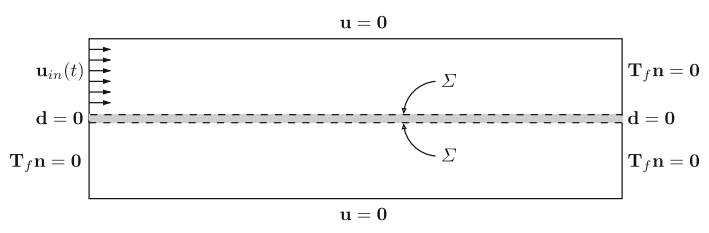

from the left boundary, where

$$
u_{\text {in }, x}(t)= \begin{cases}10 t & t \leq 0.1 \\ 0 & \text { otherwise }\end{cases}
$$

while we prescribe a homogeneous Neumann condition to the other three ends of the two compartments. The fluid-structure interface $\Sigma$, namely the upper and lower boundaries of the barrier, is free to move; see Fig. 10.

In the discrete setting, we set $\Delta t=10^{-3} \mathrm{~s}, T=0.25 \mathrm{~s}, \gamma_{v}=\gamma_{p}=10$. The fluid and structure meshes initially consist of uniform and regular triangles consisting of 1100 elements $(h=0.025 \mathrm{~cm})$ and 400 elements $(h=0.01 \mathrm{~cm})$, respectively. Due to their intersection, polygonal elements appear. We employ the Backward Difference Formula (BDF) scheme of order 3 for the temporal discretization.

We pick the pairs of velocity and pressure spaces $\mathcal{P}^{\ell}-\mathcal{P}^{m}$, with $\ell=3$ and $m=1,2,3$, both with and without the pressure stabilization term $(4 \mathrm{~d})$. The spatial polynomial order of discrete displacement field is set equal to $\ell=3$.

In Fig. 11, we plot the pressure field at time $t=0.1 \mathrm{~s}$ for all the considered configurations. As expected, for a fixed pair of spaces $\mathcal{P}^{3}-\mathcal{P}^{m}, m=1,2,3$, the stabilized pair yields a stable and more regular pressure field compared to the not stabilized one. The not stabilized $\mathcal{P}^{3}-\mathcal{P}^{3}$ pair, Fig. $11 \mathrm{~b}$, leads to an oscillating pressure field near the inlet boundary and all along the fluid-structure interface, where elements of general shape appear. This instabilities become less evident as the pressure polynomial order decreases; see Fig. 11d and f. On the other hand, the so-called "stabilized" $\mathcal{P}^{3}-\mathcal{P}^{3}$ pair, Fig. 11a, shows some oscillations only at the corners of the inlet boundary, where we expect a lower regularity in the solution, a pressure peak and strong pressure gradients, leading to an unstable pressure field. For the stabilized $\mathcal{P}^{3}-\mathcal{P}^{2}$ and $\mathcal{P}^{3}-\mathcal{P}^{1}$ cases, see Fig. 11c and e, the pressure field does not present any noticeable oscillation. Moreover, there is no significant difference in the fluid velocity and structure displacement fields. For large times $t$, the 3-rd order BDF scheme may introduce some oscillations in the pressure field in the proximity of the fluid-structure interface due to the little dissipation of the scheme itself.

\subsection{The FSI Problem: An Elastic Membrane in a Pipe}

Here, we consider a second fluid-structure interaction problem aiming at showing that the proposed PolyDG discretization method is able to reproduce the expected dynamics of the system with a stable pressure field. More precisely, we consider a pipe filled by a viscous fluid with an immersed linear elastic membrane that blocks the flow. The pipe is represented by a fluid domain $\Omega$ of size $0.4 \mathrm{~cm} \times 0.2 \mathrm{~cm}$, while the solid domain $\Omega_{s}$ represents the elastic membrane of size $0.01 \mathrm{~cm} \times 0.2 \mathrm{~cm}$ centred in the pipe; see Fig. 12. At initial time, the system is at rest. The membrane is clamped at the pipe, i.e., $\boldsymbol{d}=\mathbf{0}$ on the upper and lower sides of $\Omega_{s}$. At the top and bottom boundaries of the fluid domain, $\boldsymbol{u}=\mathbf{0}$, while on the left and right sides we prescribe a jump in the stress, namely, $\boldsymbol{\sigma}_{f} \boldsymbol{n}=(-10,0)$ dyne $/ \mathrm{cm}$ 


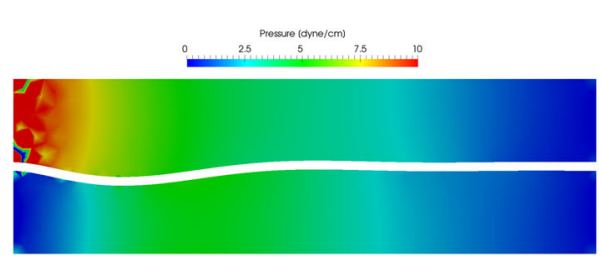

(a) $\mathcal{P}^{3}-\mathcal{P}^{3}$, stabilized

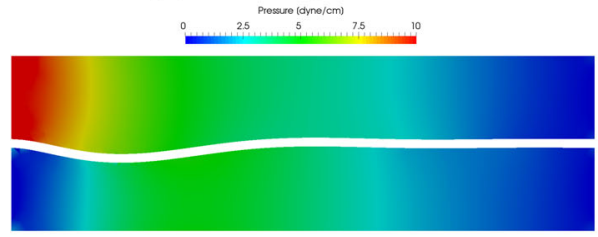

(c) $\mathcal{P}^{3}-\mathcal{P}^{2}$, stabilized
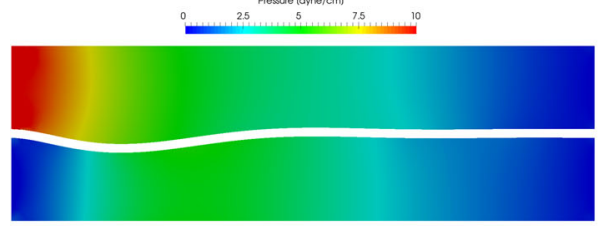

(e) $\mathcal{P}^{3}-\mathcal{P}^{1}$, stabilized

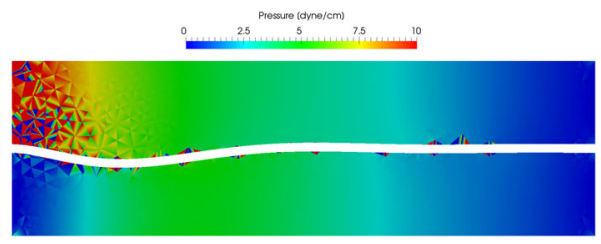

(b) $\mathcal{P}^{3}-\mathcal{P}^{3}$

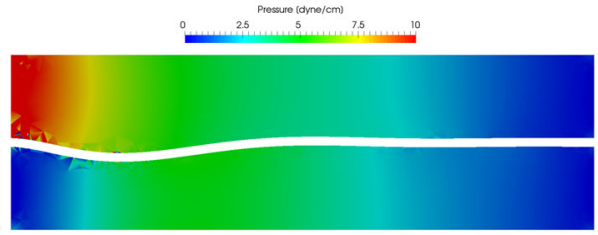

(d) $\mathcal{P}^{3}-\mathcal{P}^{2}$

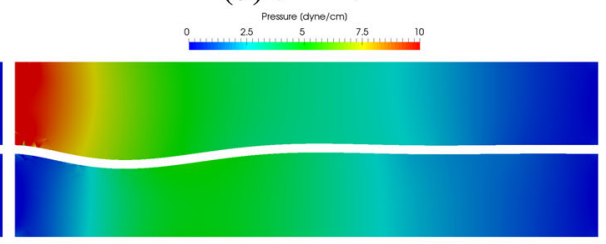

(f) $\mathcal{P}^{3}-\mathcal{P}^{1}$

Fig. 11 Pressure field at time $t=0.1 \mathrm{~s}$ for different choices of the pressure space $\mathcal{P}^{m}, m=1,2,3$, with pressure stabilization (left) and without stabilization (right). The velocity space is fixed to $\mathcal{P}^{3}$ (Color figure online)

Fig. 12 "Pipe" test case. Fluid (red) and structure (black) meshes (Color figure online)

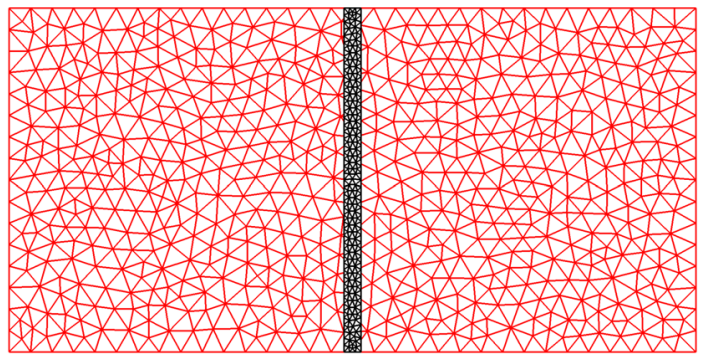

and $\boldsymbol{\sigma}_{f} \boldsymbol{n}=\mathbf{0}$, respectively. This induces oscillations in the structure, which are subsequently dumped by the viscous fluid until a steady state is reached. At the steady state, we expect a uniform pressure inside each chamber of the pipe. The fluid and structure have the following material properties: $\rho=\rho_{s}=1 \mathrm{~g} / \mathrm{cm}^{2}, \mu=0.1 \mathrm{~g} / \mathrm{s}, E=10^{4}$ dyne $/ \mathrm{cm}$ and $\nu=0.45$.

For the numerical simulation, we consider a fluid mesh consisting of 1400 elements ( $h=0.0125 \mathrm{~cm})$ and a solid mesh consisting of 400 elements $(h=0.004 \mathrm{~cm})$; see Fig. 12 . Although the meshes are initially made of regular triangles, their intersection generates elements of general shape, see Fig. 13. We consider the following discrete parameters: $\Delta t=$ $0.002 \mathrm{~s}, \gamma_{v}=\gamma_{p}=10$, and $\ell=3, m=2$ with pressure stabilization. For the time discretization, we employ the 3-rd order BDF scheme.

In Fig. 14(left), we show the configuration at the steady state, $t=1 \mathrm{~s}$. As expected, each of the two chambers of the pipe reach a uniform value of the pressure. In Fig. 14(right), we plot the $x$-displacement of the structure at its center of mass. 
Fig. 13 "Pipe" test case. Detail of the fluid mesh near the fluid-structure interface showing some polygonal elements. In blue, we highlight the possible small and anisotropic elements that may appear (Color figure online)
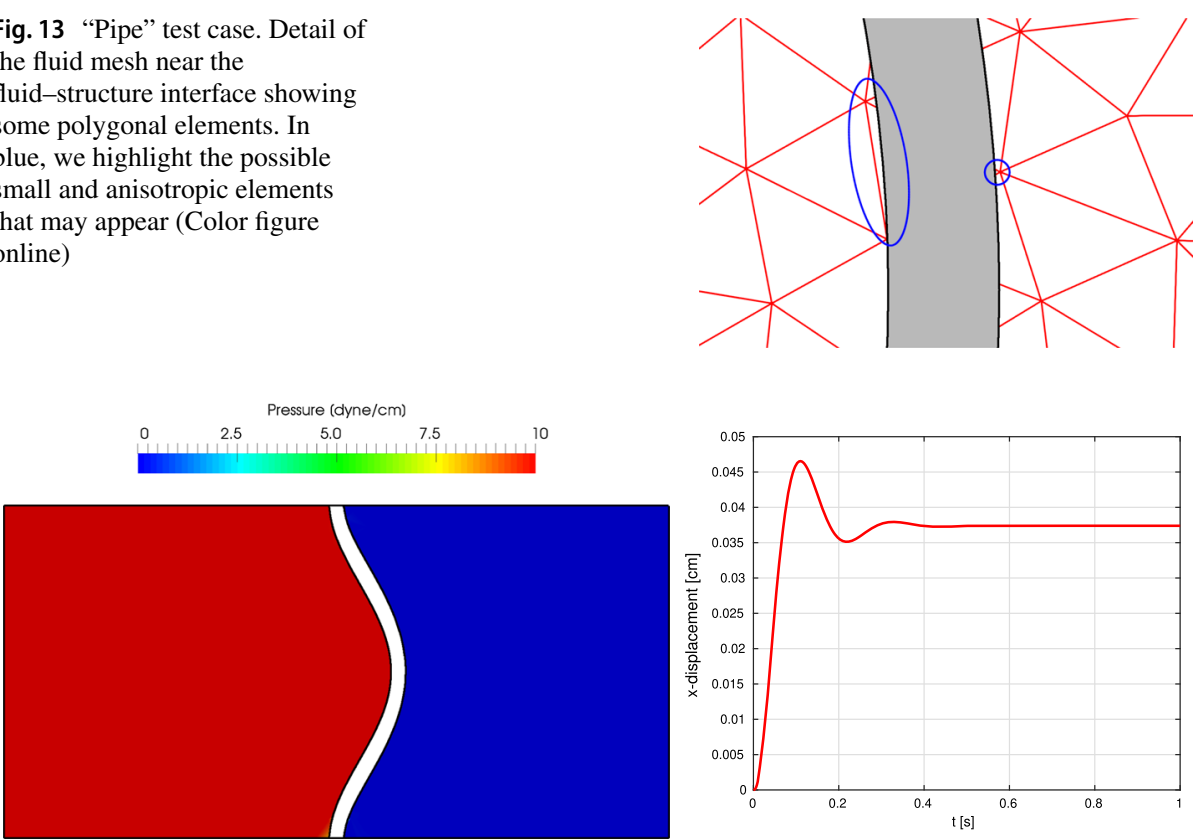

Fig. 14 "Pipe" test case. Left: pressure field and position of the structure at the steady state $(t=1 \mathrm{~s})$. Right: evolution in time of the $x$-displacement of the structure at the center of mass (Color figure online)

\section{Conclusions}

In this work, we showed the well-posedness of the discrete Stokes problem obtained via a Discontinuous Galerkin approximation on polygonal and polyhedral grids. In particular, we proved a generalized inf-sup condition that is valid for $m-\ell \leq 1$, with $\ell$ and $m$ the spatial polynomial degrees for the velocity and pressure spaces, respectively. Under suitable mesh assumptions, we proved that the discrete inf-sup constant is uniform with respect to the mesh size and presents a mild dependence with respect to the polynomial degree. Moreover, from the numerical tests, the discrete inf-sup constant seems to be uniform in much more general configurations than those covered by the theoretical analysis, indicating for example that the method is robust with respect to degenerating edges. We also proved a priori error estimate in the energy norm for the Stokes problem that is suboptimal with respect to the polynomial degree, since it inherits the suboptimality of the discrete inf-sup constant. Finally, we presented numerical examples by considering a time-dependent fluid-structure interaction problem in the case of finite displacement regime showing that the proposed PolyDG method is able to produce stable solutions.

\section{References}

1. Ager, C., Schott, B., Vuong, A.T., Popp, A., Wall, W.A.: A consistent approach for fluid-structure-contact interaction based on a porous flow model for rough surface contact. Int. J. Numer. Methods Eng. 119(13), 1345-1378 (2019)

2. Aghili, J., Boyaval, S., Di Pietro, D.A.: Hybridization of mixed high-order methods on general meshes and application to the Stokes equations. Comput. Methods Appl. Math. 15(2), 111-134 (2015) 
3. Alauzet, F., Fabrèges, B., Fernández, M.A., Landajuela, M.: Nitsche-XFEM for the coupling of an incompressible fluid with immersed thin-walled structures. Comput. Methods Appl. Mech. Eng. 301, 300-335 (2016). https://doi.org/10.1016/j.cma.2015.12.015

4. Antonietti, P.F., Cangiani, A., Collis, J., Dong, Z., Georgoulis, E.H., Giani, S., Houston, P.: Review of discontinuous Galerkin finite element methods for partial differential equations on complicated domains. In: Barrenechea, G., Brezzi, F., Cangiani, A., Georgoulis, E. (eds.) Building Bridges: Connections and Challenges in Modern Approaches to Numerical Partial Differential Equations, pp. 281-310. Springer, Cham (2016)

5. Antonietti, P.F., Facciolá, C., Houston, P., Mazzieri, I., Pennesi, G., Verani, M.: High-order discontinuous Galerkin methods on polyhedral grids for geophysical applications: seismic wave propagation and fractured reservoir simulations. In: Di Pietro, D.A., Formaggia, L., Masson, R. (eds.) Polyhedral Methods in Geosciences. SEMA-SIMAI Springer Series. Springer, Cham (2020)

6. Antonietti, P.F., Giani, S., Houston, P.: $h p$-version composite Discontinuous Galerkin methods for elliptic problems on complicated domains. SIAM J. Sci. Comput. 35(3), A1417-A1439 (2013)

7. Antonietti, P.F., Mazzieri, I.: High-order discontinuous Galerkin methods for the elastodynamics problem on polygonal and polyhedral meshes. Comput. Methods Appl. Mech. Eng. 342, 414-437 (2018)

8. Antonietti, P.F., Verani, M., Vergara, C., Zonca, S.: Numerical solution of fluid-structure interaction problems by means of a high order Discontinuous Galerkin method on polygonal grids. Finite Elem. Anal. Des. 159, 1-14 (2019)

9. Babuška, I., Suri, M.: The $h-p$ version of the finite element method with quasi-uniform meshes. ESAIM Math. Model. Numer. Anal. 21(2), 199-238 (1987)

10. Babuška, I., Suri, M.: The optimal convergence rate of the $p$-version of the finite element method. SIAM J. Numer. Anal. 24(4), 750-776 (1987)

11. Bassi, F., Botti, L., Colombo, A., Di Pietro, D.A., Tesini, P.: On the flexibility of agglomeration based physical space discontinuous Galerkin discretizations. J. Comput. Phys. 231(1), 45-65 (2012)

12. Beirão Da Veiga, L., Canuto, C., Nochetto, R.H., Vacca, G.: Equilibrium analysis of an immersed rigid leaflet by the virtual element method (2020). arXiv preprint arXiv:2007.09130

13. Boffi, D., Brezzi, F., Fortin, M.: Mixed Finite Element Methods and Applications. Springer Series in Computational Mathematics, vol. 44. Springer, Berlin (2013)

14. Boffi, D., Gastaldi, L.: A fictitious domain approach with Lagrange multiplier for fluid-structure interactions. Numer. Math. 135(3), 711-732 (2017)

15. Borazjani, I.: Fluid-structure interaction, immersed boundary-finite element method simulations of bioprosthetic heart valves. Comput. Methods Appl. Mech. Eng. 257, 103-116 (2013)

16. Borker, R., Huang, D., Grimberg, S., Farhat, C., Avery, P., Rabinovitch, J.: Mesh adaptation framework for embedded boundary methods for computational fluid dynamics and fluid-structure interaction. Int. J. Numer. Methods Fluids 90(8), 389-424 (2019)

17. Bouaanani, N., Renaud, S.: Effects of fluid-structure interaction modeling assumptions on seismic floor acceleration demands within gravity dams. Eng. Struct. 67, 1-18 (2014)

18. Braess, D., Schwab, C.: Approximation on simplices with respect to weighted Sobolev norms. J. Approx. Theory 103(2), 329-337 (2000)

19. Brezzi, F.: On the existence, uniqueness and approximation of saddle-point problems arising from Lagrangian multipliers. Publications mathématiques et informatique de Rennes S4, 1-26 (1974)

20. Burman, E., Delay, G., Ern, A.: An unfitted hybrid high-order method for the Stokes interface problem. HAL Id: hal-02519896 (2020)

21. Burman, E., Fernández, M.A.: An unfitted Nitsche method for incompressible fluid-structure interaction using overlapping meshes. Comput. Methods Appl. Mech. Eng. 279, 497-514 (2014). https://doi.org/10. 1016/j.cma.2014.07.007

22. Burman, E., Fernández, M.A., Frei, S.: A Nitsche-based formulation for fluid-structure interactions with contact. ESAIM Math. Model. Numer. Anal. 54(2), 531-564 (2020)

23. Burman, E., Frei, S., Massing, A.: Eulerian time-stepping schemes for the non-stationary Stokes equations on time-dependent domains (2019). arXiv preprint arXiv:1910.03054

24. Cangiani, A., Dong, Z., Georgoulis, E.H.: $h p$-version space-time discontinuous Galerkin methods for parabolic problems on prismatic meshes. SIAM J. Sci. Comput. 39(4), A1251-A1279 (2017)

25. Cangiani, A., Dong, Z., Georgoulis, E.H.: $h p$-version discontinuous Galerkin methods on essentially arbitrarily-shaped elements (2019)

26. Cangiani, A., Dong, Z., Georgoulis, E.H., Houston, P.: $h p$-version discontinuous Galerkin methods for advection-diffusion-reaction problems on polytopic meshes. ESAIM Math. Model. Numer. Anal. 50(3), 699-725 (2016)

27. Cangiani, A., Dong, Z., Georgoulis, E.H., Houston, P.: hp-Version Discontinuous Galerkin Methods on Polygonal and Polyhedral Meshes. Springer, Cham (2017) 
28. Cangiani, A., Georgoulis, E.H., Houston, P.: $h p$-version discontinuous Galerkin methods on polygonal and polyhedral meshes. Math. Models Methods Appl. Sci. 24(10), 2009-2041 (2014)

29. Cockburn, B., Kanschat, G., Schötzau, D., Schwab, C.: Local discontinuous Galerkin methods for the Stokes system. SIAM J. Numer. Anal. 40(1), 319-343 (2002)

30. Court, S., Fournié, M.: A fictitious domain finite element method for simulations of fluid-structure interactions: the Navier-Stokes equations coupled with a moving solid. J. Fluid. Struct. 55, 398-408 (2015)

31. Di Pietro, D.A., Ern, A.: Discrete functional analysis tools for discontinuous Galerkin methods with application to the incompressible Navier-Stokes equations. Math. Comput. 79(271), 1303-1330 (2010)

32. Di Pietro, D.A., Ern, A.: Mathematical aspects of discontinuous Galerkin methods. Mathématiques \& Applications, vol. 69. Springer, Heidelberg (2012). https://doi.org/10.1007/978-3-642-22980-0

33. Di Pietro, D.A., Ern, A., Linke, A., Schieweck, F.: A discontinuous skeletal method for the viscositydependent Stokes problem. Comput. Methods Appl. Mech. Eng. 306, 175-195 (2016)

34. Donea, J.: An arbitrary Lagrangian-Eulerian finite element method for transient dynamic fluid-structure interaction. Comput. Methods Appl. Mech. Eng. 33, 689-723 (1982)

35. Dumbser, M., Fambri, F., Furci, I., Mazza, M., Serra-Capizzano, S., Tavelli, M.: Staggered discontinuous Galerkin methods for the incompressible Navier-Stokes equations: spectral analysis and computational results. Numer. Linear Algebra Appl. 25(5), e2151 (2018)

36. Ern, A., Guermond, J.L.: Theory and Practice of Finite Elements. Applied Mathematical Sciences, vol. 159. Springer-Verlag, New York (2004). https://doi.org/10.1007/978-1-4757-4355-5

37. Ern, A., Guermond, J.L.: Finite Elements. II-Galerkin Approximation, Elliptic and Mixed PDEs. Texts in Applied Mathematics, vol. 73. Springer, Cham (2021). https://doi.org/10.1007/978-3-030-56923-5

38. Fedele, M., Faggiano, E., Dede, L., Quarteroni, A.: A patient-specific aortic valve model based on moving resistive immersed implicit surfaces. Biomech. Model. Mech. 16(5), 1779-1803 (2017)

39. Gerstenberger, A., Wall, W.A.: An extended finite element method/Lagrange multiplier based approach for fluid-structure interaction. Comput. Methods Appl. Mech. Eng. 197(19), 1699-1714 (2008)

40. Ghosh, R.P., Marom, G., Bianchi, M., D'souza, K., Zietak, W., Bluestein, D.: Numerical evaluation of transcatheter aortic valve performance during heart beating and its post-deployment fluid-structure interaction analysis. Biomech. Model. Mechanobiol. 19, 1725-1740 (2020)

41. Girault, V., Rivière, B., Wheeler, M.: A discontinuous Galerkin method with nonoverlapping domain decomposition for the Stokes and Navier-Stokes problems. Math. Comput. 74(249), 53-84 (2005)

42. Glowinski, R., Pan, T.W., Hesla, T.I., Joseph, D.D., Periaux, J.: A fictitious domain approach to the direct numerical simulation of incompressible viscous flow past moving rigid bodies: application to particulate flow. J. Comput. Phys. 169(2), 363-426 (2001)

43. Griffith, B.E.: Immersed boundary model of aortic heart valve dynamics with physiological driving and loading conditions. Int. J. Numer. Methods Biomed. Eng. 28(3), 317-345 (2012)

44. Hairer, E., Nørsett, S.P., Wanner, G.: Solving Ordinary Differential Equations I. Nonstiff Problems. Springer-Verlag, Berlin (1993)

45. Hansbo, P., Larson, M.G.: Discontinuous Galerkin methods for incompressible and nearly incompressible elasticity by Nitsche's method. Comput. Methods Appl. Mech. Eng. 191(17-18), 1895-1908 (2002)

46. Hron, J., Turek, S.: A monolithic FEM/multigrid solver for an ALE formulation of fluid-structure interaction with applications in biomechanics. In: Bungartz, H.J., Schafer, M. (eds.) Fluid-Structure Interaction, pp. 146-170. Springer, Berlin (2006)

47. Kamakoti, R., Shyy, W.: Fluid-structure interaction for aeroelastic applications. Prog. Aerosp. Sci. 40(8), 535-558 (2004)

48. Lehrenfeld, C., Olshanskii, M.: An Eulerian finite element method for PDEs in time-dependent domains. ESAIM: Mathematical Modelling and Numerical Analysis 53(2), 585-614 (2019)

49. Massing, A., Larson, M.G., Logg, A., Rognes, M.E.: A Nitsche-based cut finite element method for a fluid-structure interaction problem. Commun. Appl. Math. Comput. Sci. 10(2), 97-120 (2015)

50. Mittal, R., Iaccarino, G.: Immersed boundary methods. Annu. Rev. Fluid Mech. 37(1), 239-261 (2005). https://doi.org/10.1146/annurev.fluid.37.061903.175743

51. Picelli, R., Ranjbarzadeh, S., Sivapuram, R., Gioria, R., Silva, E.: Topology optimization of binary structures under design-dependent fluid-structure interaction loads. Struct. Multidiscip. Optim. 62, 1-16(2020)

52. Schötzau, D., Schwab, C., Stenberg, R.: Mixed $h p$-FEM on anisotropic meshes II: hanging nodes and tensor products of boundary layer meshes. Numer. Math. 83(4), 667-697 (1999)

53. Schötzau, D., Schwab, C., Toselli, A.: Mixed $h p$-DGFEM for incompressible flows. SIAM J. Numer. Anal. 40(6), 2171-2194 (2002)

54. Schötzau, D., Schwab, C., Toselli, A.: Stabilized $h p$-DGFEM for incompressible flow. Math. Models Methods Appl. Sci. 13(10), 1413-1436 (2003) 
55. Schwab, C.: $p$ - and $h p$ - Finite Element Methods: Theory and Applications in Solid and Fluid Mechanics. Clarendon Press, Oxford (1998)

56. Stein, E.M.: Singular Integrals and Differentiability Properties of Functions. Princeton University Press, Princeton, NY (1970)

57. Stenberg, R., Suri, M.: Mixed $h p$ finite element methods for problems in elasticity and Stokes flow. Numer. Math. 72(3), 367-389 (1996)

58. Talischi, C., Paulino, G.H., Pereira, A., Menezes, I.F.M.: Polymesher: a general-purpose mesh generator for polygonal elements written in Matlab. Struct. Multidiscip. Optim. 45(3), 309-328 (2012)

59. Tavelli, M., Dumbser, M.: Arbitrary high order accurate space-time discontinuous Galerkin finite element schemes on staggered unstructured meshes for linear elasticity. J. Comput. Phys. 366, 386-414 (2018)

60. Tello, A., Codina, R., Baiges, J.: Fluid structure interaction by means of variational multiscale reduced order models. Int. J. Numer. Methods Eng. 121(12), 2601-2625 (2020)

61. Terahara, T., Takizawa, K., Tezduyar, T.E., Bazilevs, Y., Hsu, M.C.: Heart valve isogeometric sequentiallycoupled FSI analysis with the space-time topology change method. Comput. Mech. 65, 1-21 (2020)

62. Tezduyar, T.E., Sathe, S.: Modelling of fluid-structure interactions with the space-time finite elements: solution techniques. Int. J. Numer. Methods Fluids 54(6-8), 855-900 (2007)

63. Toselli, A.: $h p$ discontinuous Galerkin approximations for the Stokes problem. Math. Models Methods Appl. Sci. 12(11), 1565-1597 (2002)

64. Toselli, A., Schwab, C.: Mixed $h p$-finite element approximations on geometric edge and boundary layer meshes in three dimensions. Numer. Math. 94(4), 771-801 (2003)

65. Beirão da Veiga, L., Lovadina, C., Vacca, G.: Divergence free virtual elements for the Stokes problem on polygonal meshes. ESAIM Math. Model. Numer. Anal. 51(2), 509-535 (2017)

66. Wiresaet, D., Kubatko, E.J., Michoski, C.E., Tanaka, S., Westerink, J.J., Dawson, C.: Discontinuous Galerkin methods with nodal and hybrid modal/nodal triangular, quadrilateral, and polygonal elements for nonlinear shallow water flow. Comput. Methods Appl. Mech. Eng. 270, 113-149 (2014)

67. Xu, D., Kaliviotis, E., Munjiza, A., Avital, E., Ji, C., Williams, J.: Large scale simulation of red blood cell aggregation in shear flows. J. Biomech. 46(11), 1810-1817 (2013)

68. Ye, X., Zhang, S.: A conforming discontinuous Galerkin finite element method for the Stokes problem on polytopal meshes. Int. J. Numer. Methods Fluids 93(6), 1913-1928 (2021)

69. Zhang, L.T., Gay, M.: Immersed finite element method for fluid-structure interactions. J. Fluid Struct. 23(6), 839-857 (2007)

70. Zonca, S., Antonietti, P.F., Vergara, C.: A polygonal discontinuous Galerkin formulation for contact mechanics in fluid-structure interaction problems. Commun. Comput. Phys. 30(1), 1-33 (2021)

71. Zonca, S., Vergara, C., Formaggia, L.: An unfitted formulation for the interaction of an incompressible fluid with a thick structure via an XFEM/DG approach. SIAM J. Sci. Comput. 40(1), B59-B84 (2018)

Publisher's Note Springer Nature remains neutral with regard to jurisdictional claims in published maps and institutional affiliations. 